\title{
Schutz in der Exposition, Schutz für die Exposition - Wie man in transdisziplinären und transformativen Forschungsformaten mit Ungewohntem und erhöhter Aufmerksamkeit umgeht
}

Katharina Köglberger, Raphael Dietz, Charlotte Eller, Felix M. Piontek, Marius Albiez \& Thomas Potthast ${ }^{1}$

\section{$1 \quad$ Einleitung}

Transdisziplinäres und transformatives ${ }^{2}$ Forschen bietet viele Chancen und Vorteile. So zeichnet sich transdisziplinäres Forschen dadurch aus, dass neben Wissenschaftler(inne)n verschiedener Disziplinen auch Praxisakteure ${ }^{3}$ unterschiedlicher Hintergründe substantiell am Forschungsprozess mitwirken (Defila und Di Giulio 2018a, S. 33). Durch den Einbezug von Praxisakteuren kann eine höhere Anschlussfähigkeit der Forschung an das praktische Handeln in der Gesellschaft erzielt werden. Die unterschiedlichen Akteure erweitern anhand des gegenseitigen Austauschs ihren methodischen Werkzeugkasten sowie ihren Erkenntnishorizont.

1 Lead Autor(inn)en: Katharina Köglberger und Raphael Dietz, die anderen in Reihenfolge der Beispiele im Text.

2 Die Autor(inn)en möchten einen Beitrag zur Methodik transdisziplinärer Forschung leisten, die auch transformativ vorgehen kann, aber nicht muss. Diese doppelte Ansprache wird im Beitrag an den Stellen, an denen dies zutrifft, durch Klammersetzung ausgedrückt: ,transdisziplinär (und transformativ)'. An Stellen, an denen der transformative Aspekt allerdings im Vordergrund steht (z. B. Kapitel 4), wird auf die Klammersetzung verzichtet.

3 ,Akteur' bezeichnet ein handelndes Individuum. ,Akteursgruppe' bezeichnet mehrere Individuen, die aus dem gleichen Kontext (also z. B. Wirtschaft) stammen. Die Akteure werden nach der Funktion bezeichnet, in der sie am Forschungsprojekt mitwirken. Die Definition erfolgt nach den Sektoren des 3-Kreise-Modells von Seebacher et al. (2018): Als ,Wissenschaftler(innen)` werden dem Wissenschaftssystem Angehörige 
Um diese Vorteile und Chancen ausschöpfen zu können, ist es notwendig, sich zugleich den damit verbundenen Herausforderungen zu stellen und sich auch auf „Neues einzulassen“ (Jahn 2008, S. 33). Das Arbeiten über das angestammte Arbeitsumfeld hinaus kann beinhalten, dass die Beteiligten sich unter (kritischer) Beobachtung durch andere Akteure Neuem stellen, indem sie für die eigene Tätigkeit unübliche Aufgaben oder ungewohnte Rollen übernehmen. Vor allem bei Forschungsprojekten, die neben einem transdisziplinären auch einen transformativen Ansatz verfolgen, kommt hinzu, dass dies oft nicht nur unter Beobachtung durch andere Akteure im Projekt oder dessen Umfeld, sondern auch unter erhöhter Aufmerksamkeit der Öffentlichkeit erfolgt. So kann es beispielsweise vorkommen, dass Wissenschaftler(innen) im Rahmen eines transdisziplinären (und transformativen) Forschungsprojekts zum ersten Mal in ihrer beruflichen Laufbahn auf einer Bürger(innen)versammlung ihr Projekt vorstellen und gegen ein ablehnendes oder möglicherweise sogar aufgebrachtes Publikum verteidigen müssen. Während dies für erfahrene Moderator(inn)en eine Routinesituation darstellt, kann es für die Wissenschaftler(innen) eine ungewohnte Situation sein, in der sie sich einer Exposition im Sinne des vorliegenden Beitrags ausgesetzt sehen.

Die Autor(inn)en verstehen Exposition hier wie folgt: Die Akteure sind bei der Durchführung einer Tätigkeit im Rahmen eines transdisziplinären (und transformativen) Forschungsprojekts einer erhöhten Aufmerksamkeit durch Dritte ausgesetzt und zwar in einem Bereich, in dem sie das so nicht kennen. Dies stellt für sie eine ungewohnte Situation dar, zu deren Bewältigung sie nicht einfach auf ihre professionellen Routinen zurückgreifen können. Ungewohnt können für den betroffenen Akteur sowohl die durchzuführende Tätigkeit als auch die dabei erfahrene Aufmerksamkeit sein. Von Exposition wird hier gesprochen, wenn mindestens einer der beiden Aspekte zutrifft. Die Exposition ist häufig eine Notwendigkeit, der sich die Akteure zur Durchführung des geplanten Forschungsprojekts stellen müssen. Sie kann aber zusätzlich einen Mehrwert für das Projekt

bezeichnet, die in dieser Funktion am Forschungsprojekt teilnehmen. Alle weiteren am Forschungsprojekt Beteiligte, die nicht als Angehörige des Wissenschaftssystems im Projekt mitwirken, werden als ,Praxisakteure' bezeichnet. ,Wirtschaftliche Praxisakteure' bezeichnet Akteure, die als Vertreter(innen) eines Wirtschaftsunternehmens am Forschungsprojekt teilnehmen. ,Praxisakteure der öffentlichen Verwaltung ${ }^{`}$ bezeichnet Akteure, die als Vertreter(innen) der öffentlichen Verwaltung am Forschungsprojekt teilnehmen. ,Zivilgesellschaftliche Praxisakteure` bezeichnet Akteure, die als Vertreter(innen) einer zivilgesellschaftlichen Organisation oder als Individuen am Forschungsprojekt teilnehmen. Diese Bezeichnung fasst aus Gründen der Übersichtlichkeit Akteure zusammen, die verschiedenen Sektoren wie „lokale Gemeinschaft“, „organisierte Zivilgesellschaft“ oder „Kultur“ zugeordnet werden können (so in Abb. 1 in Seebacher et al. 2018, S. 156). 
oder die Akteure mit sich bringen. Um mögliche nachteilige Auswirkungen einer Exposition zu vermeiden oder möglichst gering zu halten, bedarf es Vorkehrungen, die ausreichend Schutz vor diesen Auswirkungen bieten. Dies gilt sowohl für den Schutz der betroffenen Akteure gegenüber der Öffentlichkeit als auch innerhalb des Projekts. Solche Schutzvorkehrungen können beispielsweise Fortbildungsmaßnahmen sein, die über die üblichen Aufgaben der Akteure hinausgehen und entsprechend bei der Projektplanung und Finanzierung zusätzlich zu berücksichtigen sind.

Bisherige Ansätze, die eine Reflexion von Erfahrungen in transdisziplinären (und transformativen) Forschungsprojekten erlauben, thematisieren beispielsweise Methoden der Prozessgestaltung (z. B. Bergmann et al. 2010; Defila und Di Giulio 2018b; McDonald et al. 2009) oder Managementaufgaben, die bei solchen Projekten wahrgenommen werden müssen (z. B. Defila et al. 2006; Pohl und Hirsch Hardorn 2006; Schophaus et al. 2004). Andere fokussieren auf unterschiedliche Phasen, die in Projekten unterschieden werden können (z. B. Jahn 2008; Jahn et al. 2012), oder auf Qualitätskriterien und Evaluation (z. B. Bergmann et al. 2005; Boix Mansilla et al. 2006; Defila und Di Giulio 1999; Klein 2006). Auch Lang et al. (2012) beschäftigen sich mit idealtypischen Gestaltungsprinzipien in transdisziplinären Forschungsprojekten sowie den damit möglicherweise einhergehenden Herausforderungen. Aus der Exposition resultierende Herausforderungen bleiben allerdings in allen diesen Ansätzen unberücksichtigt. Der vorliegende Beitrag möchte mit dem Thema der Exposition einen alternativen Zugang bieten, über Erfahrungen in transdisziplinären (und transformativen) Forschungsprojekten zu reflektieren. Die Reflexion über Expositionen kann in der Vorbereitung auf ein Projekt oder in der Vorbereitung auf einzelne Aufgabenbereiche eines Projekts wie etwa der Öffentlichkeitsarbeit oder öffentlichen Interventionen von Nutzen sein. Eine solche Reflexion erlaubt es, frühzeitig ein Bewusstsein dafür zu erzeugen, welche ungewohnten Situationen für wen auftreten könnten. Dieses Wissen hilft zunächst bei der Abwägung, ob jemand bereit ist, sich auf die entsprechenden Situationen einzulassen. Sollte dies der Fall sein, ermöglicht die Reflexion, sich schon im Vorfeld effektiver auf den Umgang mit Expositionen vorzubereiten sowie die nötigen Ressourcen einzuplanen. Die Reflexion hilft, mögliche negative Folgen ${ }^{4}$, wie etwa die Überforderung von Akteuren, frühzeitig zu erkennen und somit vorausschauend Schutzvorkehrungen ergreifen zu können. Diese Schutzvorkehrungen können entweder auf die Vermeidung bzw. Verringerung der negativen Folgen oder aber auf den Umgang mit ihnen abzielen.

4 Negative Folgen spielen in das Thema Erfolg und Misserfolg hinein. Für eine fundierte Auseinandersetzung mit Risiko, Erfolg und Misserfolg in transdisziplinären (und transformativen) Forschungsprojekten siehe Gonser et al. 2019 (in diesem Band). 
Im Beitrag werden anhand der in den beteiligten BaWü-Labs ${ }^{5}$ gesammelten Erfahrungen verschiedene Expositionen aufzeigt, von denen die Autor(inn)en vermuten, dass sie bei vergleichbaren Settings auch in anderen transdisziplinären (und transformativen) Forschungsprojekten auftreten können. Wie die Ausgestaltung von Schutzvorkehrungen aussehen kann, wird im Beitrag exemplarisch aufgezeigt. Wichtig für die Entscheidung, ob es sich lohnt, sich zu exponieren, ist auch das Wissen über mögliche positive Auswirkungen. Diese sammelt und beleuchtet der Beitrag deshalb ebenfalls, wobei von ,Mehrwert' gesprochen wird, wenn positive Auswirkungen direkt mit den beschriebenen Expositionen einhergehen, und von ,positiven Effekten', wenn sie den jeweiligen Schutzvorkehrungen entspringen. Dabei gilt, dass sich die beiden zusammenhängenden Aspekte nicht immer eindeutig trennen lassen. Zudem besteht nicht der Anspruch, dass der Mehrwert oder die positiven Effekte hier völlig neue Aspekte beschreiben. Der Zweck liegt lediglich in der Zusammenführung und Zuordnung zu den jeweiligen Expositionen.

Grundlage für die Entfaltung der Expositionen sind die in den BaWü-Labs gesammelten Erfahrungen der Autor(inn)en. Deshalb werden die Expositionen schwerpunktmäßig, aber nicht ausschließlich, aus der Perspektive der Wissenschaftler(innen) betrachtet. Zur Erweiterung der Perspektive wurde an zwei Zeitpunkten im Schreibprozess von verschiedenen Praxisakteuren eine Rückmeldung eingeholt - zur ersten Fassung des Beitrags sowie zu einer späteren Fassung. Die Rückmeldungen dienten erstens der Auswahl der dargelegten Expositionen. Zweitens konnten auf diese Weise Aussagen über Expositionen, die Praxisakteure betreffen, validiert werden.

Der Beitrag ist in sechs Kapitel gegliedert. Nach der Einleitung (Kapitel 1) werden in den Kapiteln 2 bis 5 verschiedene Expositionen, die in transdisziplinären (und transformativen) Forschungsprojekten vorkommen können, dargelegt. $\mathrm{Zu}-$ nächst werden Expositionen behandelt, die sich durch das Forschungsdesign und den Forschungsgegenstand ergeben können (Kapitel 2), gefolgt von solchen, die aus einer verstärkten Öffentlichkeitsarbeit entstehen können (Kapitel 3). Anschließend wird auf Expositionen eingegangen, die sich durch Öffentliche Interventionen (Kapitel 4), sowie solche, die sich durch die Integration von Lehre in das Forschungsprojekt (Kapitel 5) ergeben können. Kapitel 6 gibt einen zusammenfassenden Überblick und reflektiert, wie eine Betrachtung mithilfe der Expo-

5 Das Ministerium für Wissenschaft, Forschung und Kunst Baden-Württemberg fördert(e) seit 2015 in zwei Förderlinien 14 sogenannte Reallabore, die im Folgenden als ,BaWü-Labs' bezeichnet werden (s. auch http://www.reallabore-bw.de, zugegriffen am 20.05.2019). Reallabore sind ,ein Forschungsformat, in dem transdisziplinär geforscht wird und gleichzeitig ein expliziter transformativer Anspruch verfolgt wird" (Defila und Di Giulio 2018c, S. 9). 
sitionsthematik sowohl für die einzelnen Akteure als auch für das Projektmanagement von Nutzen sein kann. Zudem werden mögliche Weiterentwicklungen des Expositionsthemas mit punktuellen Anknüpfungen an die zu Beginn des Beitrags dargelegten bisher gängigen Reflexionszugänge aufgezeigt.

Die Kapitel 2-5 gliedern sich in Abschnitte (2.1, 2.2 etc.), welche sich mit einzelnen Expositionen befassen. Diese Abschnitte wiederum sind anhand einer gleichbleibenden Struktur aufgebaut: Zunächst gibt ein kurzer Text einen Überblick, welche Expositionen behandelt werden. Dann werden die Expositionen durch ein Beispiel aus einem BaWü-Lab illustriert. Im Anschluss an das Beispiel folgt die allgemeine Beschreibung der daran sichtbar werdenden Expositionen. Hierbei werden Antworten auf die folgenden Fragen gegeben:

- Was ist die Ursache für die Expositionen?

- In welchen ungewohnten Tätigkeiten und/oder in welcher Aufmerksamkeit drücken sich die Expositionen aus und wer exponiert sich gegenüber wem?

- Zu welchem Zeitpunkt im Projekt kommen die Expositionen zum Tragen?

- Welche möglichen negativen Folgen können sich aus den Expositionen ergeben?

Nach der Beantwortung dieser Fragen werden Schutzvorkehrungen dargelegt, wie mit den zuvor beschriebenen Expositionen umgegangen werden kann. Hierbei wird insbesondere darauf eingegangen, wann und was durch wen getan werden muss, um diese Schutzvorkehrungen wirkungsvoll zu gestalten. Abschließend beleuchtet jeder Abschnitt auch den Mehrwert, der durch die beschriebenen Expositionen entsteht, sowie die positiven Effekte, die mit den dazu passenden Schutzvorkehrungen einhergehen.

\section{Expositionen durch das Forschungsdesign und den Forschungsgegenstand}

Um sowohl Wissenschaftler(innen) aus verschiedenen Disziplinen als auch Praxisakteure aus verschiedenen Praxisfeldern in der transdisziplinären (und transformativen) Forschung zu integrieren, ist oft ein besonderes Forschungsdesign notwendig, das beispielsweise spezielle Formen der Kommunikation innerhalb des Projektteams ${ }^{6}$, zwischen den beteiligten Akteuren untereinander oder gegen-

6 ,Projektteam‘ beschreibt jene Personen, die für Konzeption und Steuerung des Forschungsprojekts verantwortlich sind, sowie jene, die sich besonders intensiv an den Aktivitäten des Projekts beteiligen. Entsprechend dem 3-Kreise-Modell von Seebacher et al. (2018) sind dies Personen des „Kernbereichs“ und des ,inneren Akteurskreises“. 
über Externen mit sich bringt. Damit geht oft ein neues, meist ungewohntes Rollenverständnis für die Wissenschaftler(innen) einher, da sie die Kommunikation begleiten und steuern und dabei ungewohnte Aufgaben zur Gestaltung des Kommunikationsprozesses wahrnehmen müssen.

Transdisziplinäre (und transformative) Forschungsprojekte können es beispielsweise mit sich bringen, dass nicht nur das Vorgehen früher als sonst üblich transparent dargestellt werden muss, sondern sogar die Anonymität von Personen oder Institutionen, die Gegenstand der Forschung sind, aufgehoben werden muss. Weiterhin widmet sich transdisziplinäre und insbesondere transformative Forschung häufig auch politisch und gesellschaftlich sensiblen Themen, die zumeist schon von vorneherein mit einer hohen Aufmerksamkeit belegt sind. Daraus ergeben sich Expositionen für die Wissenschaftler(innen) wie auch für die Praxisakteure oder Institutionen. Im Folgenden werden die Expositionen, die durch das Gestalten von nicht anonymisierter Forschung sowie durch das Forschen in gesellschaftlich und politisch sensiblen Kontexten auftreten können, erläutert.

\subsection{Gestalten von nicht anonymisierter Forschung}

Bereits zu Beginn der Durchführung eines transdisziplinären (und transformativen) Forschungsprojekts kann es sinnvoll sein, die Anonymität von Fallbeispielen aufzuheben, um eine Transparenz des Forschungsprozesses nach außen ${ }^{7}$ herzustellen. Daraus resultieren Expositionen für Wissenschaftler(innen) und Praxisakteure.

\section{Beispiel BaWü-Lab SRB: Forschen an und mit Schulen bei Aufhebung der Anonymität}

Für die Forschung im Reallabor STADT-RAUM-BILDUNG - Reallabor für die nachhaltige Planung von Bildungslandschaften und die Integration von Aus- und Umbauten von Schulgebäuden („,BaWü-Lab SRB“) (s. auch Steckbrief im Anhang zu diesem Buch) wurden einzelne Sekundarschulen als beispielhafte Schulen (,Next Practice') ausgewählt; diese Schulen waren Praxispartner im Projekt. Ziel war es, aus den Erfahrungen der bereits stattgefundenen oder anstehenden strukturellen und physisch-räumlichen Transformation an diesen Schulen und deren Umgebung zu lernen, um diese Erkenntnisse wiederum auf andere Schulen und deren Umgebung anwenden zu können. Diese ,Next Practice'-Beispiele wurden nicht anonymisiert, wie es aus forschungsethischen Gründen üblich wäre, sondern wurden namentlich, inklusive Standortnennung, dargestellt. Dadurch war es auch für Personen, die nicht am BaWü-Lab beteiligt waren, transparent, welche Schulen als Beispielschulen dienten. Aufgrund der Vielzahl an Schulen, die als

7 ,Außen' beschreibt jene Personen, die nicht oder nur punktuell an der Forschung beteiligt sind. Entsprechend dem 3-Kreise-Modell von Seebacher et al. (2018) sind dies Personen des ,äußeren Akteurskreises“" oder der ,umgebenden Welt“. 
Beispielschulen infrage kamen, war es wichtig, die Auswahl dieser Beispielschulen zu begründen. Ausgewählt wurden ausschließlich Schulen einer bestimmten Schulart (sog. Gemeinschaftsschulen), die schon seit einigen Jahren ein besonderes pädagogisches Konzept verfolgten. Gleichzeitig spielten deren Schulgebäude und Standorte eine wesentliche Rolle bei der Auswahl. Gesucht wurden anhand dieser drei Aspekte wenige, aber möglichst verschiedene Beispielschulen, um ein breites Spektrum abzudecken. Durch die Transparenz der Auswahlkriterien und die Aufhebung der Anonymität der Beispielschulen konnten Personen, die nicht oder nur punktuell am Forschungsprojekt beteiligt waren, den Forschungsprozess und getroffene Entscheidungen nachvollziehen sowie den Stand der Forschung und erste Erkenntnisse bezogen auf die konkreten Fälle mitverfolgen.

Aufgrund der fehlenden Anonymität wurde also eine Art Bühne erzeugt. Auf dieser Bühne wurden nun nicht nur die Forschenden, sondern auch die Vertreter(innen) der gewählten ,Next Practice'-Schulen exponiert. Die Exposition der Schulen führte dazu, dass diese eine gewisse Prominenz erhielten. Es handelte sich um Schulen, die meist ungewöhnliche pädagogische Konzepte verfolgten. Da sie gleichzeitig als ,Next Practice definiert wurden, führte das beispielsweise bei Skeptiker(inne)n gegenüber diesen pädagogischen Konzepten dazu, dass sie ihre Kritik hierzu direkt kundtaten. Dies hatte rege Diskussionen bei öffentlichen Symposien des BaWü-Labs zur Folge. Die Aufhebung der Anonymität der Schulbeispiele ermöglichte aber auch, dass die Schulen tatsächlich als Vorbilder wirkten, da sich andere Schulen dort direkt Rat für den eigenen Transformationsprozess holen konnten. Einem Rechtfertigungszwang bereits zu Beginn und während der laufenden Forschung ausgesetzt zu sein, war sowohl für die Praxispartner als auch für die Wissenschaftler(innen) eine ungewohnte Situation. Für letztere bezog sich dies vorwiegend auf die Auswahlkriterien und Datenerhebungsmethoden.

Das Mitwirken der Vertreter(innen) der Beispielschulen sowohl bei der Generierung als auch bei der Auswertung der erhobenen Daten zur eigenen Schule führte zu punktgenauen Analysen und möglichen Lösungsansätzen. Die erzeugten Ergebnisse stellten, weil sie so spezifisch zugeschnitten waren, eine Art Kompensation für die Exposition der Vertreter(innen) der Beispielschulen dar. Sie wurden von den Beteiligten als großer Mehrwert für die weitere Praxisarbeit wahrgenommen.

Für transdisziplinäre (und transformative) Forschung kann es sinnvoll (oder sogar unvermeidlich) sein - entgegen forschungsethischer Gepflogenheiten - Fallbeispiele, Interviewpartner oder Ähnliches offenzulegen und somit deren Anonymität aufzuheben. Dadurch wird nicht nur Transparenz innerhalb des Projektteams hergestellt, sondern auch nach außen gegenüber Praxisakteuren und sogar Externen $^{8}$, die nur punktuell oder eben auch gar nicht an der Forschung beteiligt sind.

8 ,(Projekt)extern' bezeichnet in Abgrenzung zum Begriff ,außen“ nur jene Personen, die laut 3-Kreise-Modell von Seebacher et al. (2018) außerhalb der Kreise, also in der umgebenden Welt, stehen und sich somit nicht bewusst an Aktivitäten des Forschungsprojekts beteiligen. 
Daraus kann ein forschungsethischer Konflikt entstehen, da nun die beforschten Institutionen und/oder Personen für alle transparent sind. Eine solche Transparenz führt zu einer Exposition der Wissenschaftler(innen) und der beteiligten Praxisakteure.

Wissenschaftler(innen) exponieren sich gegenüber Kolleg(inn)en der Wissenschaft mehr als bei den meisten Forschungsprojekten üblich. Durch die Aufhebung der Anonymität und die stärkere Transparenz des Forschungsprozesses müssen die Wissenschaftler(innen) bereits zu Beginn des Forschungsprojekts oder jedenfalls früh im Prozess - also nicht erst nach Abschluss der Forschung beispielsweise ihre wissenschaftlichen Kriterien oder gewählten Methoden rechtfertigen.

Die Praxisakteure exponieren sich gegenüber Praxis und Wissenschaft. Sie müssen ihren Sonderstatus gegenüber anderen Akteuren aus der Praxis rechtfertigen und sich Kritiker(inne)n stellen. Gleichzeitig exponieren sie sich gegenüber der Wissenschaft, da sie sich deren Analyse und Bewertung aussetzen. Dies geschieht natürlich bei jeder Forschung. Bei einer Aufhebung der Anonymität geschieht dies jedoch in besonderer Weise, da dadurch zum Beispiel auch eventuell kritische Befunde direkt mit dem öffentlich bekannten Fallbeispiel in Verbindung gebracht werden können.

Durch die laufende inhaltliche und konzeptionelle Transparenz entsteht folglich eine öffentliche Bewertungs- und Angriffsfläche. Wissenschaftler(innen) und Praxisakteure finden sich dadurch in einer ungewohnten Situation wieder.

Eine Schutzvorkehrung für die Praxisakteure bietet das Format der transdisziplinären Forschung an sich, da die Praxisakteure, wenn sie zugleich Praxispartner sind, gleichberechtigt mitforschen und dadurch aktiv und direkt bei der Prozessgestaltung und der Produktion der Ergebnisse mitwirken. Sie können so nicht nur den Status quo gemeinsam mit den Wissenschaftler(inne)n reflektieren und bewerten, sondern auch bei der Darstellung der Inhalte nach außen aktiv mitwirken, so dass sie sich auf eine mögliche Konfrontation durch Kritiker(innen) vorbereiten können. Dies ermöglicht das Erzielen von passgenauen Forschungsergebnissen, die direkt von den Praxispartnern verwertet oder angewandt werden können. Dies wiederum kann jedoch zu einer weiteren Exposition der Wissenschaftler(innen) führen, wenn aufgrund der Mitwirkung der Praxispartner bei den Veröffentlichungen der Ergebnisse die Wissenschaftlichkeit der Befunde infrage gestellt wird.

Einen Mehrwert der beschriebenen Transparenz und Aufhebung der Anonymität stellt die Möglichkeit der direkten Kontaktaufnahme oder ,Besichtigung ' der Fallbeispiele dar. Diese können sogar als Vorbilder wahrgenommen werden und das Initial für Transformationen anderer Orte, Objekte oder Sachverhalte darstellen, so dass sie durch die Exposition transformative Wirkung erzeugen können. 
Darüber hinaus wird die Forschung durch die Aufhebung der Anonymität der Fallbeispiele ,greifbar', so dass vor allem abstrakte Inhalte leichter auch an Externe vermittelt werden können.

\subsection{Forschen in gesellschaftlich und politisch sensiblen Kontexten}

Herausforderungen eines transdisziplinären (und transformativen) Forschungsprojekts können durch das Agieren in gesellschaftlich und politisch sensiblen Kontexten aufgrund starker Emotionen der betroffenen Akteursgruppen und von Vorbehalten untereinander verstärkt werden. Sie münden dadurch punktuell in Expositionen einzelner Personen oder Gruppen.

\section{Beispiel BaWü-Lab SRB: Transformative Forschung im Kontext von Kindern und Ju- gendlichen}

Das BaWü-Lab SRB war an verschiedenen Sekundarschulen unterwegs. Die Schulgemeinschaft, bestehend aus Kollegium, Schülerschaft, Eltern und Bildungspartnern ${ }^{9}$, der Schulträger (die Kommune) sowie das Gebäude selbst und das umgebende Quartier bildeten den Forschungskontext. Die Schule sowie deren konzeptionelle und räumliche Transformation stellten den Forschungsgegenstand dar.

Gerade der Kontext von Kindern und Jugendlichen ist besonders sensibel, denn diese genießen ein hohes Maß an Aufmerksamkeit und Schutz in unserer Gesellschaft. Bei der Zusammenarbeit mit den Akteuren vor Ort (z. B. bei der Durchführung von Workshops) wurden die verschiedenen Interessengruppen mit ihren zum Teil konträren Haltungen deutlich. Diese Haltungen waren häufig mit starken Emotionen verbunden, was eine sachliche Diskussion erschwerte. So lagen die Interessen der Eltern vorwiegend auf der Behütung sowie auf einer erfolgreichen Schulkarriere ihres Kindes, was zur Formulierung entsprechender Forderungen gegenüber dem Lehrpersonal oder auch gegenüber dem Schulträger führte. Der Fokus der Lehrkräfte lag auf guten Lern- und Arbeitskonditionen, damit sowohl sie selbst als auch die Kinder und Jugendlichen optimale Bedingungen zum Lehren und Lernen vorfänden. Der Schulträger verfolgte meist übergreifende Interessen, wie die Quartiersentwicklung oder die Ermittlung von perspektivischen Schüler(innen)zahlen.

Damit die gemeinsame Arbeit in den Workshops möglich war und zur Entwicklung von gemeinsam getragenen Lösungsansätzen führen konnte, mussten beispielsweise die Lehrkräfte Abstand nehmen von ihrer eigenen Perspektive der Pädagogik und Didaktik und sich in das fremde Themenfeld der Architektur und Stadtplanung hineindenken, um ihre eigenen Interessen für alle verständlich formulieren zu können. Dazu mussten

9 ,Bildungspartner' sind Personen außerhalb des Lehrer(innen)kollegiums, die das pädagogische Angebot der Schule ergänzen, wie beispielsweise Sozialpädagog(inn)en oder Vertreter(innen) von Unternehmen. 
Vorbehalte gegenüber den anderen Beteiligten, beispielsweise gegenüber dem Schulträger, abgelegt werden, um sachlich argumentieren zu können. Gleichzeitig mussten Wissenslücken offen angesprochen und gemeinsam diskutiert und geklärt werden. Dies führte zu einer Exposition der Lehrkräfte gegenüber den anderen Beteiligten. Dasselbe galt für alle Beteiligten in unterschiedlichem Maße. Da der Schulträger vor allem bei der baulichen Transformation einer Schule die wichtigste Rolle innehat, war es für die zuständigen Vertreter(innen) dieses Akteurs besonders schwierig, wenn sie sich durch Wissenslücken gegenüber den anderen Beteiligten exponierten.

Um eine produktive Kooperation zwischen den Vertreter(inne)n der verschiedenen Akteursgruppen sicherzustellen, mussten die Wissenschaftler(innen) zwischen den jeweils verschiedenen Interessen vermitteln. Sie übernahmen die ungewohnte Rolle der Moderation oder gar die der Mediation, um mögliche Missverständnisse frühzeitig zu klären und eventuell aufkeimende Konflikte zu lösen. Sie agierten unparteiisch und unterstützten die Akteursgruppen dabei, jenseits von potenziellen Konflikten oder gegensätzlichen Interessen gemeinsamen Boden zu suchen und zu finden. Das Einnehmen dieser ungewohnten Rolle führte meist zu einer Exposition gegenüber den lokalen Praxisakteuren.

Zusätzlich exponierten sich die Wissenschaftler(innen) durch das Forschungsthema und den Forschungsgegenstand gegenüber der Politik, beispielsweise gegenüber dem Gemeinderat. Denn durch die Betrachtung von Gemeinschaftsschulen wurden diese als zukunftsfähige und ,gute' Schulart definiert. Dies wurde jedoch von den politischen Lagern, abhängig von den lokalen Gemengelagen, kontrovers diskutiert. Das Projektteam agierte also nicht nur im sensiblen Kontext von Kindern und Jugendlichen, sondern zudem im Spannungsfeld politischer Lager. Je nach lokalen politischen Mehrheiten in einer Kommune oder nach Prägungen aufgrund möglicher vorangegangener Zerwürfnisse bezüglich der Einführung von Gemeinschaftsschulen, exponierten sich die Wissenschaftler(innen) in diesen Auseinandersetzungen, so dass sie einem gewissermaßen ,politischen' Rechtfertigungszwang ausgesetzt waren. Um diesem vorzubeugen, wurden mehrere Termine im Gemeinderat vorgesehen. Diese fanden vor Beginn der Kooperation statt, um das Vorhaben zu erläutern und gemeinsam zu diskutieren. Eventuelle Einwände wurden aufgegriffen und die Planung gegebenenfalls angepasst. Direkt nach Abschluss wurden die Ergebnisse dem Gemeinderat vorgestellt und gemeinsam diskutiert. Die Anzahl der Termine variierte je nach Kooperationspartner.

Das Forschen in gesellschaftlich und politisch sensiblen Kontexten kann zweierlei Expositionen erzeugen: erstens Expositionen aufgrund gesellschaftlicher Spannungsfelder und zweitens Expositionen aufgrund politischer Polaritäten. Aus dem gesellschaftlichen Kontext können sich starke Interessenlagen ergeben, die konträr sein können und aufgrund der Sensibilität mit einer überaus starken Haltung und Emotion vertreten werden können. Das wiederum kann eine sachliche Kommunikation erschweren und Nährboden für Missverständnisse erzeugen. Begeben sich Wissenschaftler(innen) in dieses Spannungsfeld und erarbeiten mit Praxisakteuren Lösungsansätze, wie im vorangegangenen Beispiel beschrieben, führt dies zu Expositionen aller Beteiligten. 
Praxisakteure müssen sich nicht nur in für sie fremde Themengebiete einarbeiten, sondern exponieren sich gegenüber den jeweils anderen Interessengruppen aufgrund von fehlendem Wissen. Denn um ein gesamtheitliches Bild auf ein System zu erhalten, muss das Wissen aller Beteiligten zusammengeführt werden. Dafür wiederum ist es erforderlich, zuerst das spezialisierte Wissen von allen zu identifizieren. Dadurch werden aber auch Wissenslücken Einzelner hinsichtlich dieses Systems für alle offensichtlich. Zusätzlich kann es individuell auch an Expertise und Erfahrung mangeln, was im Prozess ebenfalls zu einer Exposition führen kann. Diese für transdisziplinäre Forschung übliche Herausforderung kann in einem gesellschaftlich sensiblen Kontext aufgrund der mitschwingenden Emotionen und möglicher gegenseitiger Voreingenommenheit zur Exposition werden. Je nach Gemengelage kann es einzelnen Praxisakteuren besonders schwerfallen, Wissenslücken oder fehlende Expertise einzugestehen, zu kommunizieren und sich damit den anderen Praxisakteuren gegenüber zu exponieren. Besonders betroffen sind davon meist Akteure mit einer besonderen Rolle im Transformationsprozess. Im vorangegangenen Beispiel wäre das der Schulträger, da er bei der Finanzierung eines Schulumbaus die entscheidende Schlüsselrolle innehat und deshalb die Situation für seine Rolle als unangemessen wahrnimmt.

Die Wissenschaftler(innen) exponieren sich erstens dadurch, dass sie häufig in dem beschriebenen gesellschaftlichen Spannungsfeld die für sie meist ungewohnte Rolle der Moderation oder gar Mediation (wie im Beispiel zuvor beschrieben) einnehmen. ${ }^{10}$ Zweitens führt auch der politische Kontext zu einer Exposition der Wissenschaftler(innen). Sie müssen sich aufgrund der Normativität, die durch das Setzen des Forschungsthemas erzeugt werden kann, je nach lokalen politischen Mehrheiten möglicherweise gegenüber Politiker(inne)n einzelner Fraktionen rechtfertigen. So entstehen „Episoden, in denen neben der internen Berücksichtigung von erfolgreichen Lernschritten, die zu angestrebten Lösungen beitragen, immer auch die politische Relevanz bestehender Ziele einer externen Bewertung ausgesetzt ist" (Groß et al. 2005, S. 211).

Starke Auseinandersetzungen zwischen den Praxisakteuren können mögliche negative Auswirkungen dieser Expositionen sein, sofern diese durch die Wissenschaftler(innen) nicht verhindert werden können. Eine ungünstige politische

10 Idealerweise sollte die Moderation oder Mediation eigentlich von Dritten übernommen werden, so dass kein Rollenkonflikt der Wissenschaftler(innen) entsteht, wenn diese als Moderator(inn)en oder Mediator(inn)en aktiv in den Prozess eingreifen, den sie untersuchen und experimentell transformieren. In mehreren BaWü-Labs zeigte sich jedoch, dass derartige Moderationen oder Mediationen zum einen oft spontan vonnöten waren, so dass die Wissenschaftler(innen) kurzfristig in diese Rolle schlüpfen mussten. Zum anderen konnten aufgrund fehlender Ressourcen meist keine externen Moderator(inn)en oder Mediator(inn)en eingesetzt werden. 
Gemengelage könnte zudem die Durchführung eines Teils des Projekts beziehungsweise der Datenerhebung erschweren oder gar verhindern. ${ }^{11}$

Die Schutzvorkehrung für die Exposition der Praxisakteure wird durch das Agieren der Wissenschaftler(innen) als Moderator(inn)en und Mediator(inn)en hergestellt. Somit stellt die Schutzvorkehrung für die Praxisakteure gleichzeitig die zuvor erläuterte Exposition der Wissenschaftler(innen) dar. Die Notwendigkeit von Moderations- und Mediationstätigkeiten kann sich während des gesamten Zusammenarbeitszeitraums mit den Praxisakteuren ergeben. Um diese Schutzvorkehrung gewährleisten zu können, bedarf es wiederum einer Schutzvorkehrung für die damit einhergehende Exposition der Wissenschaftler(innen) als Moderator(inn)en und Mediator(inn)en. Entsprechend können Aus- und Fortbildungsangebote für die Wissenschaftler(innen) eine mögliche Schutzvorkehrung darstellen, weil diese ermöglichen, die ungewohnte Rolle bereits im Vorfeld zu erlernen und zu trainieren. Einen Schutz der Wissenschaftler(innen) vor der Exposition gegenüber der Politik bieten Analysen der politischen Polaritäten bezogen auf den jeweiligen Kontext, in den diese sich begeben, so dass eine Vorbereitung auf kritische Diskussionen möglich ist. Eine Vorstellung und Erläuterung des Vorhabens beispielsweise im Gemeinderat wiederum kann eine mögliche Beoder Verhinderung des Vorhabens durch einzelne Politiker(innen) minimieren. Weitere Termine zur Vorstellung von Zwischenergebnissen und zur Präsentation des Ergebnisses können dies unterstützen.

Ein Mehrwert in der beschriebenen Exposition der Praxisakteure liegt - wie bei den meisten transdisziplinären Forschungsprojekten - darin, dass diese gegenseitig die jeweils anderen Sichtweisen, Interessen, Zielvorstellungen und Expertisen kennen sowie hoffentlich schätzen lernen. Im Zuge der Kooperation lernen sie außerdem den Fachjargon der anderen Akteure zu verstehen und anzuwenden. Gerade in einem sensiblen Kontext kann das Moderieren und Schlichten durch die Wissenschaftler(innen) verhärtete Konflikte lösen, so dass die dann eingeübte Zusammenarbeit die bestehenden Vorbehalte vorerst überbrücken kann. Dies bietet die Möglichkeit, dass die Zusammenarbeit der Praxisakteure gegebenenfalls über das Forschungsprojekt hinaus weitergeführt werden kann und mögliche Auseinandersetzungen aufgrund der geschaffenen gemeinsamen Kommunikationsbasis selbstständig ausgefochten werden können.

11 Weitere Ausführungen hierzu siehe Kapitel 4. 


\section{Expositionen durch Öffentlichkeitsarbeit}

An die BaWü-Labs wurde seitens des Fördergebers die Vorgabe gestellt, vom „Wissen zum Handeln zu kommen“ (MWK 2013, S. 31). Schäpke et al. (2017) sehen in solchen Anforderungen, die darin bestehen, neben der Wissensgenerierung Erkenntnisse in die ,gesellschaftliche Breite“ zu tragen, ein grundsätzliches Charakteristikum von Reallaboren. Um diese Anforderung zu erfüllen, fand in vielen BaWü-Labs ein Austausch mit der Öffentlichkeit und/oder eine Zusammenarbeit mit (Teilen) der Bevölkerung statt. Durch die breite öffentliche Sichtbarkeit entstanden für die beteiligten Akteure Expositionen. Im Folgenden werden die Expositionen erläutert, die durch das Organisieren von und Repräsentieren auf Veranstaltungen sowie durch das mediale Aufrufen für das Austesten von Innovationen entstehen.

\subsection{Organisieren von und Repräsentieren auf Veranstaltungen}

Im Rahmen von transdisziplinären (und transformativen) Forschungsprojekten kann es vorkommen, dass (Groß-)Veranstaltungen mit Teilnehmer(inne)n aus verschiedenen Akteursgruppen durchgeführt werden, in denen das Projektteam die tragende Rolle einnimmt. Organisieren, Veranstalten und Repräsentieren exponiert die am Projekt Beteiligten auf mehrfache Weise gegenüber den anderen Beteiligten und gegenüber externen Besucher(inne)n.

Beispiel BaWü-Lab Dietenheim zieht an: Organisation und Durchführung von Großveranstaltungen in Form der Messen „Dietenheim zieht an“

Das Reallabor Nachhaltige Transformation der Textilwirtschaft am Standort Dietenheim (,BaWü-Lab Dietenheim zieht an“) führte im Verlauf der Förderphase mehrere Messeveranstaltungen durch. In die Planung und Ausgestaltung waren alle Projektpartner und somit alle beteiligten Akteursgruppen (Wissenschaftler(innen), lokale Unternehmen und Verbände sowie die Stadtverwaltung und der Bürgermeister) involviert. Für alle Akteure war bereits im Vorfeld klar, dass vom Erfolg dieser Veranstaltungen viel abhing, da diese die größten nach außen hin sichtbaren Aktivitäten des BaWü-Labs waren. Besonders die kommunalen Akteure erhofften sich davon auch eine positive Außendarstellung. Durch die große Anzahl an erwarteten Besucher(inne)n und die Berichterstattung durch regionale Medien waren alle Beteiligten gegenüber einer breiten Öffentlichkeit exponiert. Eine weitere Exposition ergab sich dadurch, dass Unternehmen akquiriert wurden, bei „Dietenheim zieht an“ auszustellen. So waren alle im Projektteam vertretenen Akteursgruppen sowohl gegenüber den Bewohner(inne)n der Stadt und weiteren externen Besucher(inne)n als auch gegenüber den ausstellenden, teilweise neu gewonnenen Akteuren exponiert. Letzteres galt im Besonderen für einen Unternehmer, der einige seiner Geschäftspartner als Aussteller gewinnen konnte. 
Die Rolle als öffentlich auftretende Verantwortliche einer großen öffentlichen Veranstaltung war für die Wissenschaftler(innen) im Projektteam, anders als für einige Praxispartner, ungewohnt. Ziel der Veranstaltungen war eine positive Berichterstattung und somit ein erhöhter Bekanntheitsgrad sowohl des BaWü-Labs als auch der beforschten Konzepte nachhaltigen Textilkonsums.

Um die Wahrscheinlichkeit eines Misserfolgs zu mindern und von Beginn an viele Besucher(innen) anzuziehen, fand die erste Messe gleichzeitig mit dem etablierten Stadtfest „Dietenheim leuchtet“ statt. Bei allen Veranstaltungen wurden örtliche Vereine und Verbände integriert, um eine breite Öffentlichkeit anzusprechen und anzuziehen. In enger Absprache und Zusammenarbeit mit der kommunalen Verwaltung konnte eine Vielzahl regionaler Akteure gewonnen werden. Hinzu kamen umfangreiche Werbemaßnahmen in Form von Plakatwerbung in umliegenden Orten, Anzeigen in Zeitungen und sozialen Medien sowie Flyer. Die größtenteils positive, in Einzelfällen aber auch kritische Berichterstattung sowie die Wirksamkeit unterschiedlicher Werbekanäle wurden sowohl im Team der Wissenschaftler(innen) reflektiert als auch mit den Praxisakteuren diskutiert.

Trotz der umfangreichen Herausforderungen boten die Veranstaltungen eine hervorragende Gelegenheit, den Forschungsgegenstand des BaWü-Labs bekannter zu machen, und ermöglichten gleichzeitig, sowohl transformative Prozesse anzustoßen als auch Daten zu erheben.

Transdisziplinäre (und transformative) Forschungsprojekte können auf vielfache Weise in der Öffentlichkeit stehen. Projektinhalte und Teilprojekte werden häufig durch verschiedene Akteure nach außen repräsentiert. Dies kann in unterschiedlichen Formaten geschehen - von Informationsveranstaltungen in kleinem Rahmen für interessierte Kleingruppen bis hin zu Großveranstaltungen. Das führt häufig zu Expositionen gegenüber der Öffentlichkeit, unabhängig davon, ob nur vor einer kleinen Gruppe betroffener Anwohner(innen) präsentiert wird oder ob (über-)regionale Medien über eine Veranstaltung des Forschungsprojekts berichten. Kritische Fragen und negative Rückmeldungen können besonders für unvorbereitete und unerfahrene Akteure problematisch werden. Mit zunehmender Breite der angesprochenen Öffentlichkeit steigt zudem die Wahrscheinlichkeit, dass auch ein Publikum erreicht wird, das dem Projekt oder Teilen davon ablehnend gegenübersteht. Ebenfalls steigt die Wahrscheinlichkeit, dass Vertreter(innen) aller politischen Parteien und/oder Journalist(inn)en teilnehmen, und zwar unabhängig davon, welche Nähe oder Haltung zu den Projektthemen sie haben. Die daraus resultierende Exposition kann alle Akteure eines Forschungsprojekts betreffen und kann, abhängig von der Erfahrung und vom Hintergrund der Person, unterschiedlich stark ausfallen, andere Expositionen hingegen betreffen nur einzelne Akteursgruppen. 
Im Rahmen der BaWü-Labs übernahmen vorwiegend die Wissenschaftler(innen) die Organisation solcher Veranstaltungen und traten so nach außen hin als Verantwortliche und Ansprechpartner auf, sie exponierten sich also entsprechend. Präsentieren Wissenschaftler(innen) Ergebnisse ihrer Forschungsarbeit, bewegen sie sich in der Regel auf ihnen vertrautem Terrain. Dies ändert sich, wenn sie Veranstaltungen organisieren, die einer (mehr oder weniger breiten) Bekanntmachung des Forschungsgegenstands dienen - so zum Beispiel im Fall von „Dietenheim zieht an". Dann ist für die Wissenschaftler(innen) eine Exposition möglich, weil sie ungewohnte Tätigkeiten übernehmen und sich Kritik und Fragen aussetzen, die nicht nur das Projekt oder dessen Teile betreffen, sondern auch das Thema an sich.

Bei den Praxisakteuren exponieren sich jene Akteure, die nur in einem Teil des Forschungsprojekts involviert sind, aber beispielsweise im Rahmen von Veranstaltungen plötzlich auf Kritik zu anderen Teilen des Projekts reagieren sollen. Die Exposition ist umso unangenehmer, je mehr ihnen das nötige Hintergrundwissen beziehungsweise der Gesamtüberblick über das Projekt fehlen.

Die Exposition wirtschaftlicher Akteure kann davon abhängen, inwieweit sie mit den normativen Grundsätzen des Forschungsprojekts (z. B. Nachhaltige Entwicklung oder Gemeinwohl) im Einklang stehen, da sie durch ihre Teilnahme am Projekt an den entsprechenden Grundsätzen gemessen werden. Unternehmen können sich Kritik von außen aussetzen, wenn ihr unternehmerisches Handeln als nicht mit den Grundsätzen des Forschungsprojekts übereinstimmend wahrgenommen und ihre Teilnahme daher als unglaubwürdig eingestuft wird. Des Weiteren können Größe und Bekanntheit eines Unternehmens eine Rolle spielen, weil gerade größere Unternehmen mögliche nachteilige Folgen durch eine Exposition sowie die finanziellen Folgen (also inwiefern sich der Aufwand einer Teilnahme für sie beispielsweise finanziell lohnt) genau abwägen.

Ähnlich exponiert sich die öffentliche Verwaltung, und zwar insbesondere, wenn sie sich an der Einführung neuer Veranstaltungsformate beteiligt und sich somit hinsichtlich des Themas und der damit verbundenen normativen Grundsätze positioniert. Die durch das Projekt betroffene Bevölkerung nimmt häufig die Verwaltung als verantwortlich wahr und adressiert Kritik entsprechend (zum Thema Umgang mit Beschwerden an die Verwaltung s. Abschnitt 4.1).

Zivilgesellschaftliche Akteure schließlich können sich durch die Teilnahme an Großveranstaltungen transdisziplinärer (und transformativer) Forschungsprojekte gegenüber einer breiten Öffentlichkeit und kommunalen Akteuren exponieren. So kann beispielsweise eine Nachbarschaftsinitiative durch die Teilnahme an einer solchen Veranstaltung gleichzeitig größere Aufmerksamkeit erfahren als bisher und mehr Kritik ausgesetzt sein, als sie es gewohnt ist. 
Eine sehr grundlegende Schutzvorkehrung besteht darin, von Beginn eines transdisziplinären (und transformativen) Projekts an allen Akteuren die wissenschaftliche, praktische und gesellschaftliche Relevanz des Forschungsgegenstands aus Sicht jeder beteiligten Akteursgruppe zu erläutern und sicherzustellen, dass alle Beteiligten einen Überblick über das gesamte Projekt haben. Dadurch können alle Beteiligten jederzeit die Hintergründe erläutern. Durch ein transparentes und einheitliches Auftreten der beteiligten Akteure gegenüber Interessierten wie auch gegenüber Kritiker(inne)n kann zudem eine positive Außenwahrnehmung gefördert werden. Eine Absprache der wichtigen Positionen und eine Vergegenwärtigung möglicher Kritikpunkte sowie der Antworten darauf kann vor einer Großveranstaltung für die exponierten Akteure hilfreich sein. Kritik (aber auch Lob) aus den Medien und von Dritten wiederum sollte mit allen Beteiligten reflektiert werden, um zu verhindern, dass sich die Beteiligten abwertende Bemerkungen oder persönliche Angriffe zu sehr zu Herzen nehmen. Es sollte erörtert werden, weshalb etwas kritisiert wurde, welche Verbesserungen vorgenommen werden sollen oder ob die Kritik überhaupt eine Relevanz für das Projekt hat. Ein einheitliches Erscheinungsbild aller Teilprojekte mit entsprechendem Wiedererkennungswert kann eine weitere Schutzvorkehrung sein, indem es den Blick von den einzelnen Akteuren weg und zum Projekt hin lenkt und gleichzeitig die Zugehörigkeit zum Projekt zeigt. Eine umfangreiche Bewerbung von Großveranstaltungen wie etwa „Dietenheim zieht an“, um möglichst viele Besucher anzulocken, ist ebenfalls eine Schutzvorkehrung. Dadurch wird die Chance erhöht, dass beteiligte Akteure die Veranstaltung als Erfolg wahrnehmen. Zusätzlich können Presserundgänge und -mitteilungen die mediale Berichterstattung positiv beeinflussen.

Abhängig vom Forschungsgegenstand, Ziel und Setting eines Projekts können große Veranstaltungen einen hohen Mehrwert bieten. Wenn Forschungsprojekte das Ziel haben, die Bevölkerung zu integrieren und zu aktivieren, kann die Durchführung öffentlichkeitswirksamer Maßnahmen und Veranstaltungen schnell zu einem wesentlichen Teil der Projektarbeit werden. Durch Großveranstaltungen können auf einen Schlag viele Menschen erreicht und informiert oder zur Teilnahme an weiteren Veranstaltungen und/oder öffentlichen Interventionen (s. Kapitel 4) gewonnen werden. Durch erfolgreiche Veranstaltungen im Rahmen der BaWü-Labs wurde oft eine positive Berichterstattung in den Medien erreicht, die dazu führte, dass weitere Teilprojekte einfacher durchgeführt werden konnten. Erfahrungsgemäß waren auch viele beteiligte (z. B. kommunale) Akteure eher bereit, an weiteren Veranstaltungen mitzuwirken und sich weiter zu exponieren, wenn die Resonanz der Öffentlichkeit positiv war. Auch im Hinblick auf die Verstetigung von Teilprojekten (z. B. durch Übernahme seitens kommunaler oder zivilgesellschaftlicher Akteure) kann ein hoher Bekanntheitsgrad hilfreich sein. Schließlich wird ein positives Medienecho auch durch den Fördergeber wahrgenommen und kann möglicherweise sogar eine Weiterförderung erleichtern. 


\subsection{Mediales Aufrufen für das Austesten von Innovationen}

In transformativen (und transdisziplinären) Forschungsprojekten kann es zum Austesten von Innovationen notwendig sein, mediale Aufrufe durchzuführen, um den Bedarf zu erheben sowie die Umsetzung und Auswirkungen erproben zu können. Den Wissenschaftler(inne)n und beteiligten Praxisakteuren können dabei durch den öffentlichen Charakter des medialen Aufrufs und durch die Spielregeln der medialen Kommunikation Expositionen entstehen.

\section{Beispiel BaWü-Lab Space Sharing: Nutzer(innen)aufruf für den Pilotstandort}

Im Reallabor Space Sharing - Nutzungsintensivierung des Gebäudebestands durch Mehrfachnutzung und dynamische Programmierung („BaWü-Lab Space Sharing“) sollte getestet werden, wie Räume im Laufe eines Tages mehrfach genutzt werden können. Um den Pilotstandort im Calwerkopfbau am Rotebühlplatz in Stuttgart zu betreiben, musste zu Projektbeginn ein Nutzer(innen)aufruf an die Stuttgarter Stadtbevölkerung gestartet werden. Um ein Bild zu erhalten, für welche Nutzungen in der Stadt von wem Räume gesucht werden, musste dieser Aufruf so angelegt sein, dass potenzielle Interessierte aus einem möglichst großen Teil der Bevölkerung angesprochen wurden. Außerdem konnte nur durch vielfältige Rückmeldungen ausgetestet werden, welche Nutzungen unter welchen Bedingungen nacheinander und/oder parallel stattfinden könnten. Um Nutzer(innen) für den Standort zu finden, musste das Thema Space Sharing ansprechend und leicht verständlich sowie überzeugend von Projektbeginn an über die Medien vermittelt werden. Der Standort, der anfangs nicht im öffentlichen Bewusstsein verankert war, musste sichtbar gemacht werden.

Die am medialen Aufruf beteiligten Wissenschaftler(innen) der Fachgruppe Architektur der Staatlichen Akademie der Bildenden Künste Stuttgart (ABK Stuttgart) exponierten sich in diesem Prozess des Aufrufs vor allem gegenüber am Forschungsprojekt Interessierten und potenziellen Nutzer(inne)n sowie gegenüber den Kolleg(inn)en und der Öffentlichkeit. Der öffentliche Charakter des medialen Aufrufs ermöglichte es allen, die Arbeit der Wissenschaftler(innen) zu beobachten und von Projektbeginn an kritisch zu hinterfragen. Bei ausbleibender Resonanz hätten Vorwürfe wie unprofessionelles Vorgehen aufkommen können. Des Weiteren hätte der Pilotstandort im Calwerkopfbau nicht betrieben werden können.

Der mediale Aufruf stellte sich als komplexer, umfangreicher und langwieriger als ursprünglich geplant heraus, was zu Verzögerungen führte. Ein breit gestreuter Rückgriff auf unterschiedliche Medien war notwendig, wobei das Anliegen in der jeweils adäquaten Form bekannt gemacht werden musste. Der mediale Aufruf reichte schließlich von digitalen Formaten wie Homepage, Facebook und Ansprechpartner per E-Mail über Printmedien (Plakate, Infoflyer, Artikel in lokaler Presse) bis zur persönlichen Präsenz bei Veranstaltungen Dritter. Entscheidend für die Resonanz aus der Bevölkerung war die Entwicklung eines identitätsstiftenden Kommunikationskonzepts - ähnlich wie eine Corporate Identity $(\mathrm{Cl})$ bei Firmen - in Kooperation mit der Fachgruppe Kommunikationsdesign der ABK Stuttgart. Es bestand aus einem leicht wiedererkennbaren Logo 
(s. dazu Abb. 1), das von digitalen Medien über Printmedien bis zu Visualisierungen und Umsetzungen im Raum zur Anwendung kam, der Wortmarke "Calwerkopfbau" und einem einheitlichen Leitsystem (s. dazu Abb. 2), das bereits vor dem Gebäude, im Treppenhaus und in den Space-Sharing-Räumlichkeiten als ,physisches Handbuch' diente. Um Interessierte, die sich vom Nutzer(innen)aufruf angesprochen fühlten, die Entscheidung zur Teilnahme zu erleichtern, wurden zu Beginn der Standortbetreibung

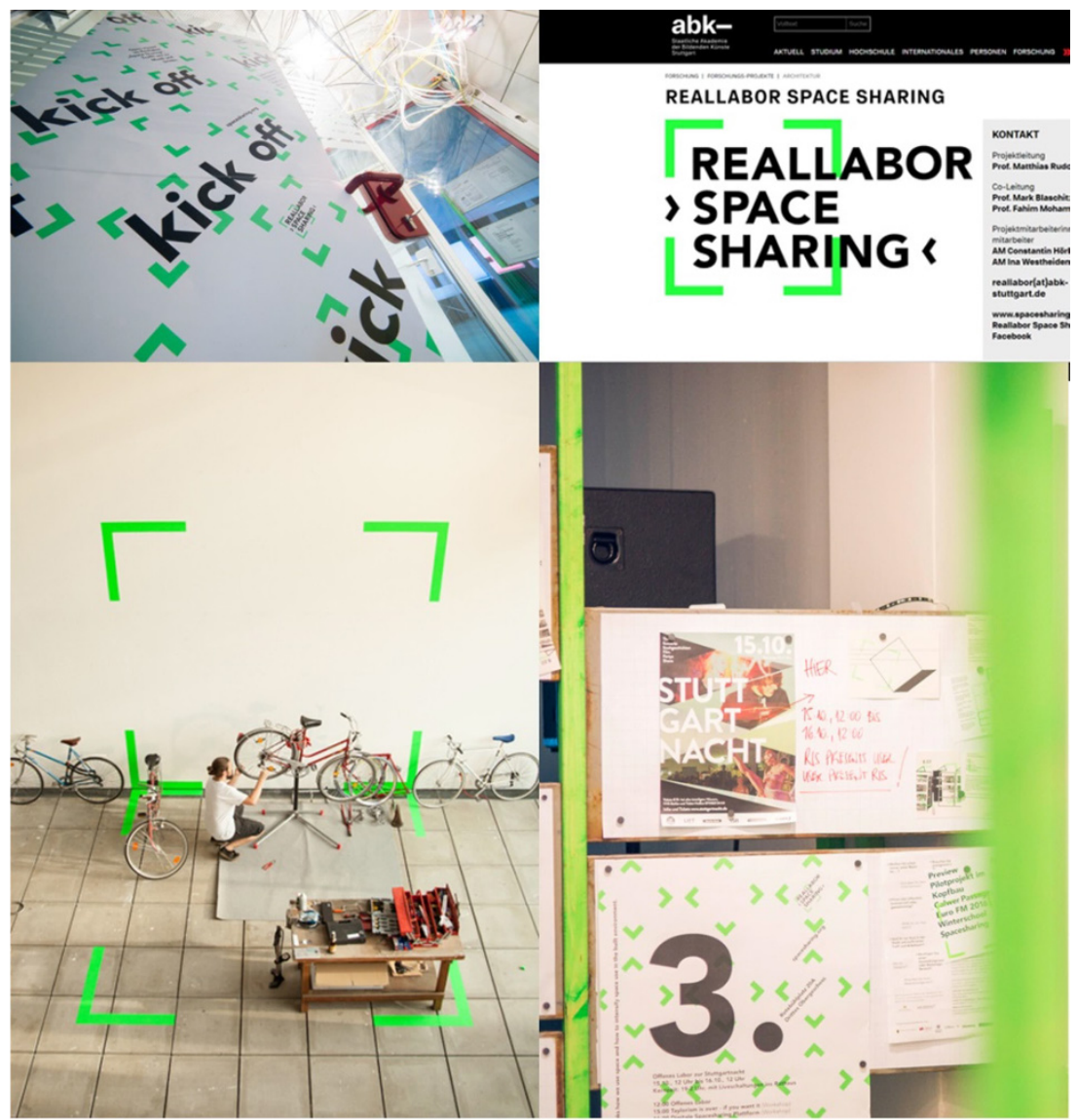

Abbildung 1: Das Logo wurde bei Plakatgestaltungen, auf der Webseite und als Umsetzung im Raum beim 24/7-Realexperiment angewendet, und die Farbe des Logos fand in der Gestaltung der Räumlichkeiten Anwendung. (C) Oben und rechts unten Reallabor Space Sharing, ABK Stuttgart; links unten Master Stegreif Spacesharing 24/7, ABK Stuttgart; Plakatgestaltung: Marlene Kehle, Erika Mai, Juliane Windbiel, Tina Zeltwanger. 
Informationsabende veranstaltet. An diesen wurde erstens ausführlich über das Projekt informiert und auf den experimentellen Charakter des Standorts hingewiesen. Zweitens gab es auch die Möglichkeit zur Diskussion sowie zum persönlichen Kennenlernen der Wissenschaftler(innen) sowie der anderen Interessierten. Diese Diskussionsveranstaltungen wurden später zum Austausch mit den Nutzer(inne)n in regelmäßigen Abständen fortgeführt. Des Weiteren erwiesen sich Multiplikatoren - z. B. Studierende oder bereits beteiligte Nutzer(innen) - als hilfreich bei der Kommunikation mit Interessierten (zu Kommunikationsstrategien im BaWü-Lab Space Sharing s. RLSS 2018, S. 39ff.).

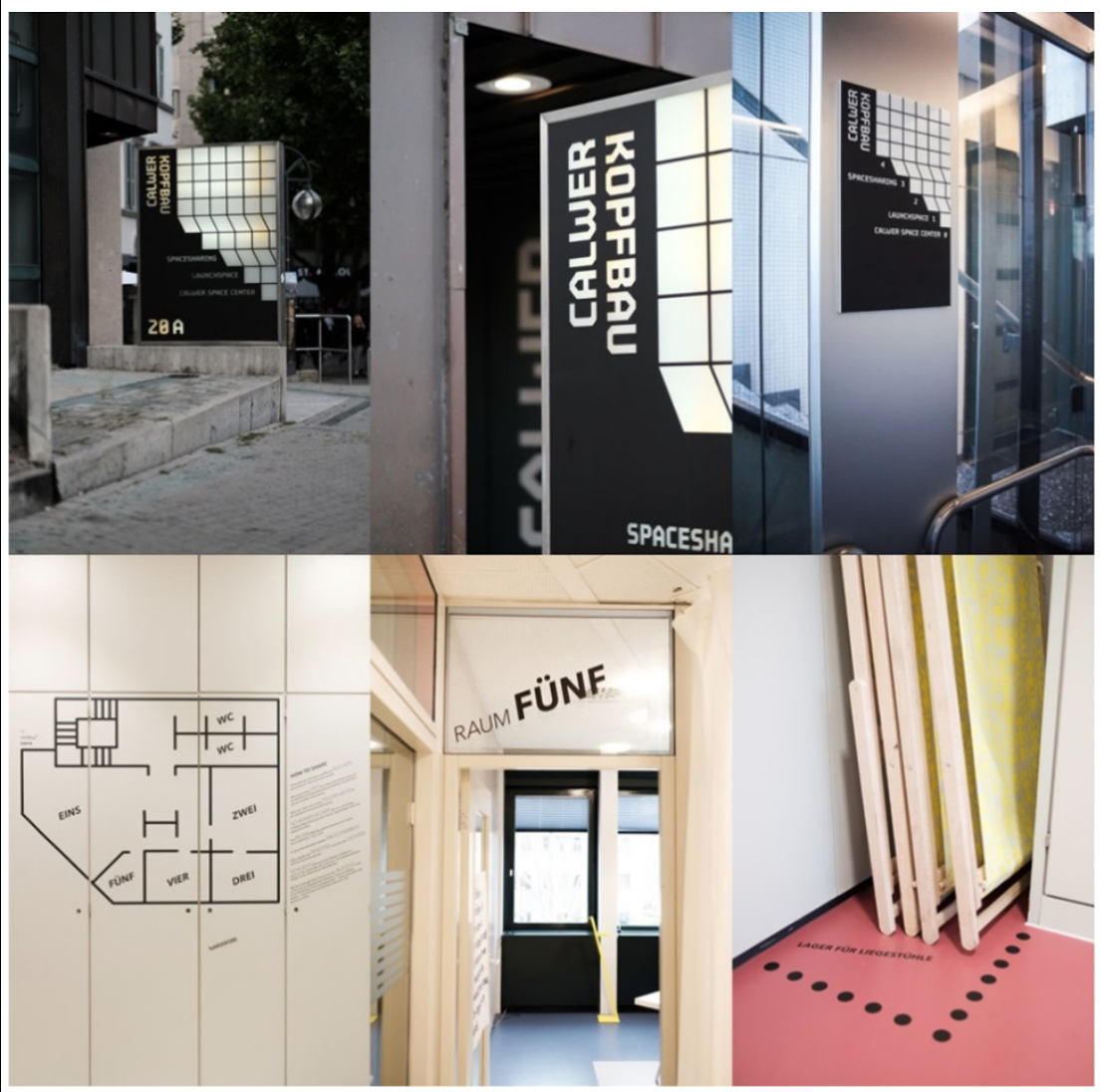

Abbildung 2: Das Leitsystem zog sich von vor dem Gebäude über Aufzug und Treppenhaus bis in die Space-Sharing-Räumlichkeiten im 3. Obergeschoss. Es bewirkte die Präsenz des Projekts im Stadtraum und diente den Nutzer(inne)n vom Eingang weg bis zum Möbel als , physisches Handbuch'. (C) Marlene Kehle, Erika Mai, Juliane Windbiel, Tina Zeltwanger. 
Für das umfassende Austesten einer Innovation (Bedarfserhebung, Erprobung der Umsetzung, Überprüfung der Auswirkungen etc.) im gesellschaftlichen Kontext kann es notwendig sein, einen medialen Aufruf durchzuführen, um den Bedarf zu erheben und Teilnehmer(innen) zu finden. Dabei können für die Wissenschaftler(innen) - sowie gegebenenfalls auch für involvierte Praxisakteure - durch den öffentlichen Charakter des medialen Aufrufs und die Spielregeln der medialen Kommunikation Expositionen entstehen.

Da die Erhebung von Bedarf und Zielpublikum der Innovation Teil der Forschungsfrage ist, kann der mediale Aufruf nicht fokussiert werden. Vielmehr muss er möglichst breit gestreut - also öffentlich über eine große Bandbreite an Medien - erfolgen. Zum Beispiel kann nicht gezielt nach 100 sportlichen Personen zwischen 50 und 60 Jahren gesucht werden, sondern es muss, wie im Beispiel des BaWü-Labs Space Sharing, möglichst die komplette Stadtbevölkerung angesprochen werden, um zu erforschen, wer wie Bedarf für die Innovation (in diesem Fall Space Sharing) hat. Zudem kann es wegen des transformativen Anspruchs notwendig sein, eine möglichst große Vielfalt gesellschaftlicher Gruppen anzusprechen. Um wissenschaftlich brauchbare Rückmeldungen aus der Bevölkerung zu erhalten, kann jedoch in der Kommunikation über die Medien die Bedarfserhebung für die Innovation nicht als Untersuchungsfrage formuliert werden. Stattdessen ist es in solchen Fällen notwendig, durch eine überzeugende Darstellung der Innovation Interesse und Bewusstsein für den Bedarf zu generieren, um mögliche und möglichst viele Interessierte zur Teilnahme zu motivieren. Es müssen also Aspekte, die erst im Laufe des Projekts nach erfolgreichem medialem Aufruf untersucht werden können, bereits zu Projektbeginn als affirmative Aussagen in der Öffentlichkeit präsentiert werden.

Die Notwendigkeit der ungerichteten und breiten Ansprache sowie die Mechanismen der medialen Kommunikation (Stichwort Vermarktung) widersprechen in einem solchen Prozess des medialen Aufrufs also den üblichen Vorgehensweisen in der Forschung. Die verantwortlichen Wissenschaftler(innen) exponieren sich erstens, weil sie mit und über die Medien arbeiten müssen. Dies entspricht nicht ihrem üblichen Aufgabenfeld und bringt eine große öffentliche Aufmerksamkeit mit sich. Die Kommunikation über die Medien ermöglicht es de facto jedem Interessierten von Beginn des medialen Aufrufs an die Arbeit im Forschungsprojekt zu verfolgen und zu kritisieren. Je nach Tätigkeitsfeld und Erfahrung betrifft dies auch am medialen Aufruf beteiligte Praxisakteure. Zweitens machen sich Wissenschaftler(innen) durch die Anpassung an die Spielregeln der medialen Kommunikation gemessen am Konsens zu wissenschaftlichen Vorgehensweisen im Wissenschaftssystem angreifbar. Mögliche negative Folgen können daher sein, dass ihnen unprofessionelles Handeln vorgeworfen wird - sowohl bezogen auf die Wissenschaftlichkeit als auch bezogen auf die Medienkommunikation. 
Außerdem ist das Austesten der Innovation vom Rücklauf auf den medialen Aufruf abhängig. Bleibt der Rücklauf aus, können folglich der Testlauf nicht wie geplant stattfinden und die damit einhergehenden Forschungsfragen nicht beantwortet werden. Erschwerend kommt hinzu, dass der Verlauf medialer Kommunikationsprozesse aufgrund komplexer Zusammenhänge und Eigendynamiken erstens schwer plan- und steuerbar sowie zweitens unzureichend analysierbar ist. Eine korrekte Analyse bei fehlendem Rücklauf, ob kein Bedarf vorhanden ist oder ob der mediale Aufruf nicht adäquat durchgeführt wurde, ist daher schwierig. Bei zu geringem Rücklauf muss mit der schon grundsätzlich schwierigen Herausforderung, das (Teil-)Projekt zur Innovation nicht wie geplant durchführen zu können, auch noch unter einer breiten Aufmerksamkeit agiert werden. Handlungsmöglichkeiten können dadurch eingeschränkt sein. Zum Beispiel kann gegebenenfalls nicht innerhalb des Projektteams nach Lösungsstrategien wie Neuausrichtung oder Ersatzmaßnahmen gesucht werden, ohne diese nach außen rechtfertigen zu müssen.

Um Wissenschaftler(innen) - sowie gegebenenfalls am Medienaufruf beteiligte Praxisakteure - nicht ungeschützt dem für sie normalerweise fremden Bereich der Medienkommunikation auszusetzen, ist es zumindest notwendig, sie für diese Aufgabe zu schulen. Als Schutzvorkehrung empfehlenswerter ist es jedoch, Akteure mit entsprechender Medienexpertise von Anfang an ins Projektteam zu integrieren. Sie können so bereits in der Projektkonzeption das notwendige Wissen einbringen, wie über welche Medienkanäle wer erreicht werden kann und dazu passende Kommunikationskonzepte entwickeln. Um erstens ein Ausbleiben des Rücklaufs auf Grund von Fehlern beim medialen Aufruf zu vermeiden und zweitens korrekte Schlüsse aus dem Rücklauf in die Bedarfsanalyse einfließen lassen zu können, empfiehlt es sich zudem, mit einem medialen Aufruf von Anfang an gleich bewusst wie mit der Wahl sämtlicher anderer Methoden umzugehen. Auch die zeitliche Komponente sollte in der Projektplanung berücksichtigt werden - so bedarf der mediale Aufruf eines ausreichend langen zeitlichen Vorlaufs für die Vorbereitung und dann für die Verbreitung über Medien und Mundpropaganda. Mediale Eigendynamiken können hierbei sowohl beschleunigend als auch verzögernd wirken. Um kritischen Stimmen sowie zweifelnden Interessierten die Möglichkeit zu geben, Zweifel und Kritik zielgerichtet zu äußern (statt ungerichtet in sozialen Medien), sollte ihnen die Möglichkeit gegeben werden, mit den betroffenen Akteuren direkt zu diskutieren. Hierzu empfehlen sich Kommunikations- und Veranstaltungsformate, auf denen ausdrücklich ein persönlicher Kontakt hergestellt werden kann - also zum Beispiel die Einrichtung einer E-Mail-Adresse, über die in kurzen Zeitfenstern Anfragen beantwortet werden, oder das Gesprächsangebot bei Veranstaltungen. Es gilt die Spanne zwischen breiter Öffentlichkeitsansprache einerseits und dem Bedürfnis nach dem persönlichen Gesprächsangebot andererseits abzudecken. 
Ein breiter medialer Auftritt zur Mitwirkung kann auch einen Mehrwert für ein Projekt darstellen. Durch die beschriebene Kommunikation des Forschungsvorhabens über verschiedene Medien entsteht die Notwendigkeit, bereits frühzeitig über die Forschungsfragen, -ziele und -methoden zu reflektieren und diese auf den Punkt gebracht für unterschiedliche Kanäle medientauglich zu formulieren. Dies kann zu einer inhaltlichen Schärfung und Fokussierung des Vorhabens beitragen, da sich alle Projektverantwortlichen über die essenziellen Aspekte einig werden müssen. Darüber hinaus kann Mehrwert durch den medialen Aufruf entstehen, wenn es gelingt, die Innovation so ansprechend zu kommunizieren, dass ein Beitrag zur Ingangsetzung des transformativen Prozesses geleistet wird. Des Weiteren können sich Interessierte, die von Seiten der Projektverantwortlichen nicht in Betracht gezogen worden waren, auf den medialen Aufruf hin melden und neue Inhalte einbringen. Schließlich kann ein positiver Effekt durch die ergriffenen Schutzvorkehrungen für die am Kommunikationsprozess beteiligten Wissenschaftler(innen) sowie Praxisakteure entstehen, indem sie sich Medienkompetenzen aneignen.

\section{Expositionen durch Öffentliche Interventionen}

Die Anforderung an transformative Forschung, gesellschaftliche Veränderungsprozesse anzustoßen, kann es mit sich bringen, dass Interventionen, beispielsweise in Form von Realexperimenten ${ }^{12}$, durchgeführt werden. Im Folgenden werden Expositionen erläutert, die aus der Durchführung von Interventionen im öffentlichen Raum entstehen. Unter solchen Interventionen werden gezielte Veränderungen des Status quo verstanden, die öffentlich sichtbar und wirksam sind und in der Regel die Genehmigung durch eine zuständige Behörde benötigen. Expositionen können durch öffentlich formulierte Kritik wie auch durch Beschwerden entstehen, die an das Projekt- bzw. Interventionsteam ${ }^{13}$ gerichtet wer-

12 Bei Realexperimenten handelt es sich um „Experimentierprozesse, die nicht in der Sonderwelt der wissenschaftlichen Laboratorien, sondern in der Gesellschaft stattfinden. [...] In Realexperimenten geht es zwar ebenso um gezielte Eingriffe und um Erkenntnisgewinn, jedoch nicht in erster Linie um Forschung und neues Wissen. [...] Da jedoch selten über die genaue Abfolge und die Erfolgsaussichtungen [sic] ein unzweifelhafter Konsens besteht, kommt der Frage entscheidende Bedeutung zu, wie man bei gegebenen Wissenslücken und Unsicherheiten dennoch zum Handeln kommen kann“ (Groß et al. 2005, S. 11).

13 Das Realexperiment, das hier als Beispiel dient, wurde in enger Kooperation zwischen Wissenschaftler(inne)n und den initiierenden Praxisakteuren durchgeführt. Da sich in diesem Prozess die Rollenverteilung nicht immer klar abgrenzen ließ und viele Aktivitäten gemeinsam geplant, durchgeführt und evaluiert wurden und da angenommen werden darf, dass dies auch in anderen Fällen so sein könnte, wird von den an der 
den. Diese Kritik kann sich auf unterschiedliche Aspekte der Intervention beziehen. Anhand des Realexperiments „Parklets für Stuttgart“ werden Expositionen beleuchtet, die durch die Kritik an Genehmigung und Durchführung von Interventionen sowie durch die Rechtfertigung für die Involvierung von projektexternen Personen entstehen.

\section{Beispiel BaWü-Lab RNM: „Parklets für Stuttgart“ - Hintergrund des Realexperiments}

Im Rahmen des Realexperiments „Parklets für Stuttgart“ (Lazarova et al. 2018) wurden über einen Zeitraum von drei Monaten an verschiedenen Standorten in der Stuttgarter Innenstadt öffentliche Parkplätze zu sogenannten Parklets ${ }^{14}$ umgestaltet. Ziel des Vorhabens war es, eine öffentliche Diskussion über Flächengerechtigkeit und die Gestaltung des öffentlichen Raums anzuregen sowie gleichzeitig Alternativen erlebbar zu machen. Initiiert wurde das Realexperiment durch zivilgesellschaftliche Praxisakteure ${ }^{15}$, deren Vorhaben in einem Ideenwettbewerb von einer transdisziplinär besetzten Jury zur Umsetzung ausgewählt wurde (zum Auswahlprozess s. Puttrowait et al. 2018). Das Reallabor für nachhaltige Mobilitätskultur („BaWü-Lab RNM“) unterstützte das Vorhaben finanziell und durch wissenschaftliche Begleitung. Daraus ergab sich ein intensiver Kooperationsprozess zwischen den zivilgesellschaftlichen und weiteren Praxisakteuren und den für das Realexperiment zuständigen Wissenschaftler(inne)n. Die Stadt Stuttgart, vertreten durch das Amt für öffentliche Ordnung, erarbeitete eine Genehmigungsgrundlage. Geplant und gebaut wurden die Parklets von Studierenden der Architektur und Stadtplanung der Universität Stuttgart im Rahmen einer eigens eingerichteten Lehrveranstaltung, die durch das Interventionsteam betreut wurde. Sie entwarfen die Parklets in Abstimmung mit lokalen Pat(inn)en, also an den Standorten anliegenden Privatpersonen oder Gewerbetreibenden, die während der Standzeit der Parklets auch für die Pflege und als Ansprechpartner, ihres' jeweiligen Parklets verantwortlich waren.

Durchführung einer solchen Intervention Beteiligten im Folgenden als ,Interventionsteam' gesprochen.

14 Als ,Parklet' bezeichnet man die räumliche Erweiterung des Gehwegs mittels einer temporären oder dauerhaften Installation, die anstelle einer Parkfläche für PKW Raum für Menschen schafft. Parklets können vielfältig gestaltet sein und unterschiedliche Funktionen wie Sitzgelegenheiten, Spielflächen oder Urban Gardening anbieten (vgl. San Francisco Planning Department 2018).

15 Dies waren drei Studierende der Architektur und Stadtplanung der Universität Stuttgart, zusammen mit weiteren zivilgesellschaftlichen Akteuren. Da die Studierenden aus privaten Motiven und außerhalb universitärer Verpflichtungen handelten, werden die Initiator(inn)en im Weiteren als zivilgesellschaftliche Praxisakteure bezeichnet. 


\subsection{Kritik an Genehmigung und Durchführung von Interventionen}

Öffentliche Interventionen können Beschwerden und öffentlich formulierte Kritik aufgrund einer inhaltlichen Ablehnung der mit der Intervention verbundenen Ziele oder der Vorgehensweise in der Umsetzung hervorrufen, wodurch sich sowohl die durchführenden Akteure als auch die genehmigenden Behörden exponiert sehen.

\section{Beispiel BaWü-Lab RNM: „Parklets für Stuttgart“ - Genehmigung und Beschwerde- management}

Das Realexperiment zog sowohl ein breites mediales Echo als auch eine öffentliche Diskussion in den sozialen Medien nach sich, wobei neben Zustimmung auch Kritik laut wurde. Zudem wurden an das Interventionsteam und die Verwaltung auch konkrete Beschwerden gerichtet. Die Beschwerden und Kommentare bewegten sich dabei auf unterschiedlichen inhaltlichen Ebenen. So wurde die Sinnhaftigkeit der Intervention generell infrage gestellt, da darin eine Verschlimmerung der Parkplatzsituation gesehen wurde. Andere kritisierten dagegen die Ästhetik oder Funktionalität einzelner Parklets. Teilweise wurde auch die Rechtmäßigkeit der Genehmigung der Intervention angezweifelt, weswegen es essenziell gewesen war, eine rechtlich nicht anfechtbare Begründung für die Genehmigung zu finden. Die meisten Beschwerden wurden direkt an die Stadtverwaltung und das Interventionsteam herangetragen. In einigen der betroffenen Stadtbezirke forderten auch die Bezirksbeiräte eine persönliche Vorstellung in einer öffentlichen Sitzung, da sie die Befürchtung äußerten, dass sie von den Bürger(inne)n auf die Intervention angesprochen und Beschwerden an sie adressiert werden würden. Für das Interventionsteam stellten diese Art und Menge der Kritik eine neue Erfahrung dar, in der sich die Mitglieder exponiert sahen. Die involvierten Vertreter(innen) der Stadtverwaltung hingegen verfügten zwar schon über eine gewisse Erfahrung mit Beschwerden, doch auch bei ihnen wurde deutlich, dass die quantitative Zunahme einen zusätzlichen Aufwand darstellte.

Dem Interventionsteam war daran gelegen, zu verhindern, dass sich die öffentliche Meinung lediglich aufgrund mangelnder Kommunikation und nicht aufgrund sachlicher Argumente gegen das Vorhaben wendete und somit eine gegebenenfalls sinnvolle Verstetigung aus sachfremden Gründen behindert würde. Zudem stand die Möglichkeit im Raum, dass die Genehmigung bei zu großem Widerstand widerrufen worden wäre, was den Abbruch des Realexperiments zur Folge gehabt hätte. Daher wurde als Schutzvorkehrung ein zwischen dem Interventionsteam und der Verwaltung gemeinsam abgestimmtes Beschwerdemanagement eingerichtet. Das Interventionsteam veröffentlichte eine eigene E-Mail-Adresse sowie die telefonischen und postalischen Kontaktdaten eines Ansprechpartners. An den Parklets selbst wurden erklärende Tafeln angebracht, die das Realexperiment erläuterten und auf Möglichkeiten zur Rückmeldung hinwiesen.

Persönlich beim Amt für öffentliche Ordnung vorgetragene Beschwerden wurden dort direkt bearbeitet. Schriftliche Beschwerden wurden beim Interventionsteam gesammelt und durch dieses beantwortet, wofür ein Verhaltenskodex vereinbart wurde. Jeder 
Person, die sich beschwerte, wurde zudem ein persönliches Gespräch angeboten. Bei konkreten Mängeln bezüglich der Gestaltung der Parklets wurde schnellstmöglich Abhilfe geschaffen (z. B. durch notwendige Reparaturen). Um die Politiker(innen) zu informieren, wurde das Vorhaben wie gewünscht durch das Interventionsteam präsentiert. Im Laufe des Realexperiments wurde zudem ein Rundgang mit interessierten Fraktionen durchgeführt, der es den Teilnehmenden ermöglichte, sich über Ergebnisse und Erfahrungen mit den Parklets auszutauschen.

Das Beschwerdemanagement war mit einem erheblichen Aufwand verbunden, doch durch das Ergreifen der beschriebenen Maßnahmen konnten ein möglicher Abbruch vermieden und das Ziel einer Diskussion über die Sache erreicht werden. Die Beschwerden wurden schließlich sogar Teil des Datenmaterials, anhand dessen diese Diskussion systematisch ausgewertet wurde.

Obwohl bei der Vorstellung des Projekts in den Bezirksbeiräten einige Fraktionen das Vorhaben inhaltlich ablehnten, zeigten sich die Politiker(innen) insgesamt dankbar über die Information, da diese sie in die Lage versetzte, Auskunft geben und Beschwerden weiterleiten zu können. Für das Interventionsteam wurde dagegen sichtbar, welche politischen Gruppierungen das Projekt unterstützten und welche es aus welchen Gründen ablehnten. Zudem lieferten die Politiker(innen) wertvolle Hinweise dazu, wie die Parklets besser in den lokalen Gemeinschaften verankert werden konnten.

Wie das Beispiel „Parklets für Stuttgart“ zeigt, kann bei einer öffentlichen Intervention mit Kritik oder konkreten Beschwerden aus der Öffentlichkeit oder der Politik gerechnet werden, die schon während der Planungsphase, vor allem aber ab Sichtbarwerdung der Intervention eintreten kann.

Durch die Durchführung der Intervention im öffentlichen Raum entsteht eine Exposition für die verantwortlichen Wissenschaftler(innen) und Praxisakteure gegenüber den Teilen der Öffentlichkeit und der Politik, die die Ziele und Inhalte sowie das Vorgehen der Intervention kritisieren. Der öffentliche Charakter kann dazu führen, dass eine große Menge an einzelnen Beschwerden das Interventionsteam erreicht. Die Menge der Beschwerden, aber auch die Art der Kritik kann für das Interventionsteam eine ungewohnte Situation darstellen, weil die Kritik nicht nur innerhalb eines fachlichen (und zumeist strukturierten) Austauschs, sondern über öffentliche Kanäle formuliert wird. Zudem konzentrieren sich die Argumente der Kritiker(innen) nicht immer auf sachliche Argumente, sondern können sogar persönliche Angriffe beinhalten. Gegenüber der Verwaltung exponiert sich das Interventionsteam durch die Verpflichtungen, wie zum Beispiel Genehmigungsauflagen, die mit der Intervention einhergehen und deren Einhaltung von der Verwaltung kritisch überwacht werden kann.

Die Erfahrung mit den Parklets zeigte, dass die Verwaltung zudem sehr auf die öffentliche Meinung achtet und in Folge dieser Meinung zusätzlichen Druck auf das Interventionsteam ausüben beziehungsweise selbst erfahrenen Druck an das 
Interventionsteam weitergeben kann. Als mögliche negative Folge der Art und Menge von Kritik und Beschwerden kann sich erstens eine inhaltliche Ablehnung der Intervention in Öffentlichkeit und Politik ergeben. Zweitens kann der Eindruck unprofessionellen Vorgehens entstehen und sich ein negatives öffentliches Meinungsbild lediglich aufgrund suboptimaler Kommunikation und Handhabe von Beschwerden entwickeln. Dieses negative Meinungsbild kann den Zielvorstellungen der Praxisakteure zuwiderlaufen und die wissenschaftlichen Akteure in ihrem Bestreben nach Wissenserwerb behindern, da kostbare Ressourcen auf das Beantworten von Beschwerden verwendet werden müssen.

Die Akteure der öffentlichen Verwaltung wiederum sehen sich gegenüber der Öffentlichkeit exponiert, da sie für die Genehmigungserteilung kritisiert und im schlimmsten Fall juristisch belangt werden können. Zusätzlich sehen sie sich dem Druck von Politiker(inne)n ausgesetzt, die eigene oder durch die Bürgerschaft an sie herangetragene Kritik am Genehmigungsprozess, wegen Fehlern in der Durchführung und Kommunikation oder an den Zielen der Intervention an die Verwaltung herantragen. Ungewohnt an dieser Situation sind für die Verwaltung vor allem der wissenschaftliche und experimentelle Kontext sowie mögliche spezifische Eigenschaften der Intervention, die das Erarbeiten und spätere Verteidigen einer Genehmigung im Vergleich zu Themen, bei denen Erfahrungen und Routinen vorhanden sind, erschweren können. Zudem kommt es zu einer quantitativen Zunahme der Beschwerden, wodurch es zu zusätzlichen Belastungen kommen kann, wenn dafür keine personellen Ressourcen zur Verfügung gestellt werden. Eine mögliche negative Konsequenz von alldem kann sein, dass die Verwaltung zukünftigen Kooperationen ablehnend gegenübersteht. Im schlimmsten Fall droht auch der vorzeitige Abbruch der Intervention durch Entzug der Genehmigung oder aufgrund einer erfolgreichen Klage.

Lokale Politiker(innen) werden von Beginn der Intervention an durch das Interventionsteam gegenüber den Bürger(inne)n exponiert, da sie von diesen auf die Intervention angesprochen werden können. Dies kann dazu führen, dass die Politiker(innen) daraufhin Druck auf die öffentliche Verwaltung ausüben, der dann an das Interventionsteam weitergegeben wird. Das Interventionsteam und die öffentliche Verwaltung sehen sich also wiederum der Reaktion durch die Politiker(innen) ausgesetzt.

Die Erfahrung des BaWü-Labs RNM zeigt schließlich, dass es kaum gelingt, eine Differenzierung zwischen einer einzelnen Intervention und dem Forschungsprojekt als Ganzes in der öffentlichen Wahrnehmung zu erreichen, weswegen die Kritik an der Intervention auch immer auf das Gesamtprojekt zurückfallen kann, wodurch sich dieses ebenfalls exponiert sieht. Eine erste Schutzvorkehrung für alle Akteure besteht darin, die Öffentlichkeit über das Projekt adäquat zu informieren, wodurch der Kritik entgegengewirkt werden kann, welche lediglich aus Unkenntnis und der ,Überraschung' über die Intervention entsteht. Eine Schutz- 
vorkehrung vor ablehnender inhaltlicher Kritik kann während der Intervention in einer aktiven, mit guten Argumenten untermauerten Beteiligung an der öffentlichen Diskussion bestehen. Hierfür können die verschiedenen Medien regelmäßig auf entsprechende Inhalte gescannt und Beiträge verfasst werden. Auch können persönliche Gespräche oder Informationsveranstaltungen vor Ort angeboten werden.

Eine weitere wichtige Schutzvorkehrung stellt ein zwischen den Akteuren der öffentlichen Verwaltung und dem Interventionsteam abgestimmtes Verfahren für den Umgang mit den Beschwerden dar, welches darlegt, welche Kommunikationskanäle zum Einreichen von Beschwerden angeboten werden, wer für die Beantwortung verantwortlich ist, welcher Verhaltenskodex für die Beantwortung gilt und wie mit Beschwerden verfahren wird, die sich nicht durch eine Antwort oder Auskunft erledigen lassen. Als Schutzvorkehrung gegen juristische Konsequenzen sollte die Verwaltung im Zuge der Planung der Intervention, wie für jede andere Maßnahme im öffentlichen Raum auch, eine juristisch sichere Grundlage für die Genehmigung finden. Als Schutzvorkehrung für lokale Politiker(innen) sind eine umfassende Information oder ein persönlicher Austausch über die Intervention mit dem Interventionsteam dienlich.

Als Mehrwert ergibt sich, dass mit der Durchführung von öffentlichen Interventionen viele projektexterne Personen in die Thematik einbezogen oder darauf aufmerksam gemacht werden sowie wissenschaftliche Fragestellungen in einem gesellschaftlichen Kontext untersucht werden können, wie es dem Wesenskern von Realexperimenten entspricht. Eine erfolgreiche Genehmigung kann einen Präzedenzfall schaffen, der es ermöglicht, vergleichbare Interventionen auch an anderen Orten durchzuführen, woraus sich ein transformativer Verstetigungseffekt ergeben kann. Positive Effekte der Schutzvorkehrungen liegen darin, dass die in den Beschwerden und der öffentlichen Diskussion enthaltenen Informationen als Daten genutzt werden können, die dazu beitragen, die mit der Intervention verbundenen Forschungsfragen zu beantworten. Werden diese analytisch ausgewertet, können sich darüber hinaus Hinweise ergeben, welche Probleme auftreten und welche Aspekte gut funktionieren. Dies kann im Sinne des „rekursiven Lernens“ (vgl. Groß et al. 2005) dazu genutzt werden, die mit der Intervention untersuchte Maßnahme zu verbessern und die Akzeptanz zu steigern. Ein weiterer positiver Effekt ergibt sich aus dem Austausch mit den Politiker(inne)n, da lokales Wissen erschlossen werden kann, das erlaubt, die Intervention noch besser auf lokale Gegebenheiten anzupassen. Bei inhaltlicher Zustimmung können die Politiker(innen) zudem als Multiplikator(inn)en für eine positive Haltung gegenüber der Intervention dienen. 


\subsection{Rechtfertigung für die Involvierung von projektexternen Personen}

Durchführende Akteure und Verantwortliche von öffentlichen Interventionen können in Kritik geraten, weil projektexterne Personen betroffen sind, ohne ihre Zustimmung zur Involvierung in ein Forschungsprojekt gegeben zu haben. Sie exponieren sich sowohl gegenüber den Betroffenen als auch im forschungsethischen Diskurs.

\section{Beispiel BaWü-Lab RNM: „Parklets für Stuttgart“ - Umgang mit Anliegenden}

Die Parklets wurden auf öffentlichen Parkplätzen errichtet, die vor allem durch Anwohner(innen) und Kund(inn)en von Gewerbetreibenden genutzt wurden. Dies führte zu einer kontroversen Diskussion, bei der die Argumente öffentlich ausgetauscht oder als direkte Rückmeldung an das Interventionsteam gerichtet wurden. Neben der zuvor beschriebenen inhaltlichen Auseinandersetzung über Sinn und Ziele der Intervention meldeten sich auch unmittelbar Betroffene zu Wort. Vor allem Anwohner(innen) beschwerten sich, die sich von den Parklets zum Beispiel durch Lärm belästigt sahen oder denen ,ihr Parkplatz' (den sie gewohnheitsmäßig für sich beanspruchten, obwohl es sich um öffentliche Flächen handelt) abhandengekommen war. Anliegende Gewerbetreibende hatten Sorge, dass aufgrund des Parkplatzmangels Kund(inn)en ausbleiben könnten. Ein anliegender Gewerbetreibender beauftragte aus diesem Grund sogar einen Anwalt, um die Rechtmäßigkeit der Genehmigung zu prüfen, da er negative Auswirkungen auf sein Geschäft fürchtete. Das Interventionsteam und die genehmigende Verwaltung sahen sich also auch hier durch Kritik und Beschwerden exponiert, allerdings bewegte sich diese Kritik nicht mehr auf der Ebene der Forschungsfrage, sondern auf der Ebene der persönlichen Betroffenheit.

Um etwaige Gefährdungen durch die Parklets auszuschließen und mögliche Störungen von Anliegenden auf ein vertretbares Maß zu beschränken, wurden als Schutzvorkehrung mit der Genehmigung entsprechende Auflagen verbunden und bei der Gestaltung der Parklets berücksichtigt. Projektexterne waren nur in einem solchen Maß Veränderungen ausgesetzt, wie es mit herkömmlichen Gestaltungsmaßnahmen vergleichbar war. Die Intervention wurde somit durch das Interventionsteam und die genehmigende Verwaltung als vertretbar eingestuft.

Der größte Teil der öffentlichen Diskussion lief über soziale Medien und die lokale Presse, worauf das Interventionsteam nur sehr begrenzt Einfluss nehmen konnte. Um die Anliegenden nicht mit der Veränderung zu überraschen, wurde vorbeugend mittels eines Einwurfschreibens und Beschilderungen an den Parkplätzen über die bevorstehende Intervention informiert. Um auch einen persönlichen Austausch zu ermöglichen, wurden verschiedene Diskussions- und Informationsformate, wie eine Podiumsdiskussion oder ein Eröffnungsrundgang, angeboten. Es zeigte sich jedoch, dass diese Angebote von den Betroffenen kaum wahrgenommen wurden. Stattdessen richteten sie ihre Beschwerden vor allem direkt an die Verwaltung, die daraufhin vom Interventionsteam und den Pat(inn)en der Parklets konkrete Nachbesserungen wie das Einhalten der Sperrstunde auf den Parklets verlangte. 
Aufgrund der Durchführung von Interventionen im öffentlichen Raum können projektexterne Personen betroffen sein, die nicht vorher um ihre Zustimmung zum Einbezug in das Forschungsprojekt gebeten werden können. So kamen im Beispiel „Parklets für Stuttgart“ Anwohner(innen) und Kund(inn)en mit den Parklets in Berührung. Dies bedeutet nicht, dass sich aus öffentlichen Interventionen eine nachteilige Auswirkung auf die Betroffenen ergeben muss, aber sie verursachen zumindest eine Veränderung der üblichen Umstände. Die ungewohnte Situation sowie gegebenenfalls nachteilige Auswirkungen können bei den Betroffenen negative Gefühle wie Irritation, Unmut oder Überforderung hervorrufen. Hierbei kann auch die persönliche Wahrnehmung einer Veränderung als individueller Nachteil eine wichtige Rolle spielen.

Infolgedessen kann es in erster Linie zu einer Exposition der die Intervention durchführenden und genehmigenden Akteure (aus Wissenschaft und Praxis) gegenüber den Betroffenen kommen, wenn diese ihre negativen Gefühle sowie persönlichen Wahrnehmungen und Standpunkte an sie adressieren. Hierbei liegt nicht die sachliche Kritik oder Beschwerde im Vordergrund (dazu s. Abschnitt 4.1), sondern die (als nachteilig empfundenen) Veränderungen der persönlichen Lebensumstände sowie die Infragestellung, warum man in ein Forschungsprojekt involviert werde.

In zweiter Linie exponieren sich auch die verantwortlichen Akteure wie Interventions- und Projektleitende, da sie das grundsätzliche Vorgehen im Forschungsprojekt, die konkrete Intervention sowie die damit einhergehenden Auswirkungen im Diskurs mit den Betroffenen rechtfertigen müssen.

Verantwortliche Wissenschaftler(innen) exponieren sich darüber hinaus gegenüber Wissenschaft und Öffentlichkeit. Laut forschungsethischem Konsens werden Personen üblicherweise über Inhalte, Methoden und Ziele eines Forschungsprojekts informiert, dann wird ihre Zustimmung zur Beteiligung eingeholt, und erst danach werden sie in das Projekt involviert. Wenn dies, wie bei Interventionen im öffentlichen Raum, nicht möglich ist, sehen sich die verantwortlichen Wissenschaftler(innen) mit einem forschungsethischen Konflikt konfrontiert. Vor allem dann, wenn Beteiligte Auswirkungen als negativ einstufen, können nicht nur das konkrete Forschungsprojekt, sondern transformative Forschungsformate per se in Kritik geraten. Negative Folgen für das einzelne Forschungsprojekt bestehen darin, dass heftige Kritik von Betroffenen oder Fachkolleg(inn)en schwerwiegende Auswirkungen auf die Durchführung des Projekts haben kann (z. B. Abbruch der Intervention, Verhinderung von darauf aufbauenden Schritten, Teilprojekten sowie Verstetigung) oder auf dessen Rezeption (z. B. Diskreditierung der Ergebnisse). 
Als vorbeugende Schutzvorkehrung sollten daher Reflexionsrunden eingebaut werden, bei denen geplante Interventionen kritisch geprüft und gegebenenfalls angepasst werden. Die Zumutbarkeit von Auswirkungen von Interventionen auf projektexterne Personen sollte besonders sorgfältig überprüft werden. Die Genehmigung durch die öffentliche Verwaltung stellt hierbei eine gute Prüfinstanz dar. Des Weiteren sollten die beteiligten Akteure vor dem Schritt in die Öffentlichkeit im Rahmen einer Reflexionsrunde projektintern über das Vorhaben und allfällige Kritikpunkte reflektieren und sich gegenseitig austauschen, um bereits vor der Umsetzung der Intervention einen Prozess des bewussten Umgangs mit ablehnenden und emotionalen Haltungen $\mathrm{zu}$ starten. Solche InformationsReflexionsrunden mögen aufgrund des Umfangs der Vorbereitungen für die Durchführung sowie dem Enthusiasmus, die Intervention nach dem oft langwierigen und umständlichen Vorbereitungsprozess (Planung, Genehmigungen, Finanzierung etc.) endlich umsetzen zu können, eine zeitliche und organisatorische Herausforderung sein. Sie ermöglichen aber, gut vorbereitet zu sein für klärende, sachliche Gespräche und Schriftverkehr, wenn während der Durchführung der Intervention oder im Nachgang ablehnende und/oder emotionale Reaktionen sowie Unverständnis von Betroffenen an die Adresse der ausführenden Akteure gerichtet werden. Es empfiehlt sich, sich bewusst darauf vorzubereiten, dass voraussichtlich nicht alle projektexternen Personen mit ihrer Involvierung in das Projekt einverstanden sein werden (bezüglich Informationsangebot vor Ort und Beschwerdemanagement s. Abschnitt 4.1). Des Weiteren sollte als Schutzvorkehrung für die Wissenschaftler(innen) daran gearbeitet werden, in der Bevölkerung wie in der Wissenschaft ein Bewusstsein dafür zu schaffen, dass der transformative Anspruch von Forschung je nach Thema und Ziel ein Wirken über die Projektgrenzen hinaus erfordern und damit zum Beispiel die Involvierung von projektexternen Personen nötig machen kann.

Aus der beschriebenen Exposition geht kein direkter Mehrwert hervor, der über den zuvor beschriebenen (s. Abschnitt 4.1), mit der Durchführung der Intervention zusammenhängenden, hinausgeht. Ein positiver Effekt aus den Schutzvorkehrungen kann für das Forschungsprojekt durch die vorausschauende Reflexion sowie den kritischen Diskurs mit projektexternen Personen entstehen, wenn diese konstruktiv zur Verbesserung der Intervention und somit zur Qualitätssteigerung im Forschungsprojekt genutzt werden. Des Weiteren kann ein gut vorbereiteter Dialog mit projektexternen Betroffenen zum transformativen Prozess beitragen, wenn es in dessen Rahmen gelingt, einen Reflexionsprozess bei den Gesprächspartner(inne)n anzustoßen und somit die Anliegen des Forschungsprojekts in die Bevölkerung hineinzutragen. 


\section{Expositionen durch die Integration von Lehre in das Forschungsprojekt}

Lehre ist neben der Forschung - idealtypisch verstanden als Einheit - die zentrale Aufgabe von Universitäten und Hochschulen; entsprechend ist sie auch für transdisziplinäre und transformative Forschungsprojekte zumindest programmatisch, oft aber auch praktisch bedeutsam. Dies kann eine große Bandbreite an Lehr- und Lernformaten betreffen, von eher fachspezifischen bis hin zu fächerübergreifenden Veranstaltungen mit Praxisakteuren. In diesen findet Exposition von Studierenden, Praxisakteuren und Lehrenden statt, sowohl gegenüber der Öffentlichkeit als auch untereinander. Die nachfolgenden Ausführungen beziehen sich auf Lehrerfahrungen aus dem Energielabor Tübingen - Potenziale, Partizipation, Perspektiven (,BaWü-Lab Energielabor Tübingen“) einschließlich der mit diesem BaWü-Lab assoziierten Lehr- und Lernaktivitäten (vgl. Albiez et al. 2018). ${ }^{16}$ Die Lehrenden waren gleichzeitig als Wissenschaftler(innen) im BaWü-Lab Energielabor Tübingen tätig. Anhand dieser Erfahrungen werden die Expositionen beleuchtet, die sich ergeben durch Erwartungen im Hinblick auf Strukturen der Wissensvermittlung und das Verhältnis von Empirie und normativer Orientierung sowie durch die Mitwirkung an transdisziplinären und transformativen Lehrveranstaltungen.

\subsection{Erwartungen im Hinblick auf Strukturen der Wissensvermittlung und das Verhältnis von Empirie und normativer Orientierung}

Lehrende exponieren sich durch das transdisziplinäre und transformative Forschungsformat Reallabor. Dies gilt sowohl für den Umgang mit Erwartungen der Studierenden zum vorhandenen Fachwissen der Lehrenden als auch für die Bestimmung des Verhältnisses zwischen ,Fakten', Werten und Normen.

Beispiel BaWü-Lab Energielabor Tübingen: Umgang mit Erwartungen zu wertorientierter Forschung in Lehrveranstaltungen

Neben fachspezifischen Seminaren, die sich beispielsweise an angehende Lehrkräfte im naturwissenschaftlichen Bereich an der Universität Tübingen richteten, wurden einzelne Lehreinheiten für angehende Ingenieur(inn)e(n) an der Universität Stuttgart angeboten. In allen Lehrveranstaltungen wurde bereits zu Beginn auf das Forschungsformat Reallabor und die damit einhergehenden transdisziplinären und transformativen For-

16 Gemeint sind hier Lehrveranstaltungen, die im Zusammenhang mit dem Forschungsprojekt „Wissenschaftliche Bildung und gesellschaftliche Verantwortung“ standen. Hierbei flossen Know-how zu transdisziplinärer Lehre sowie personelle Ressourcen aus dem BaWü-Lab Energielabor Tübingen in die Lehraktivitäten ein. 
schungsansätze eingegangen. Zudem wurde das Verhältnis zu anderen Forschungsansätzen aufgezeigt. Die Lehrenden exponierten sich zunächst durch die Breite des Gegenstands Energiewende. So herrschte bei den Studierenden teilweise die Vorstellung vor, transdisziplinäre Nachhaltigkeitswissenschaftler(innen) verfügten über disziplinäre Fachkenntnis in allen für das Thema Energiewende relevanten Feldern. Sie müssten beispielsweise zugleich Expert(inn)en für die Funktion technischer Anlagen sein, für die sozio-technische Transformation des Mobilitätssektors und für Lebenszyklusanalysen in der Umweltbilanzierung. Des Weiteren exponierten sich die Lehrenden dadurch, dass sie ausdrücklich auf die Verknüpfung von Empirie und Normen hinwiesen. Dies stand im Gegensatz zur Erwartung mancher Studierender, dass Wissenschaft rein evidenz- und faktenbasiert betrieben werden müsse. Um dieses Spannungsfeld zu thematisieren, wurden beispielsweise aktionsorientierte Lehrmethoden wie "Six Thinking Hats" von de Bono (1999) ${ }^{17}$ angepasst und genutzt. Die Studierenden versetzten sich in die Rolle von Projektmanager(inne)n und beschäftigten sich in Übernahme unterschiedlicher Sichtweisen und Diskussionstypen (quantitativ faktenbasiert, emotional, risikobewusst, synergie-/konsensorientiert) mit dem Thema Energiewende. Ziel war es, bei den Studierenden ein Bewusstsein dafür zu fördern, dass im Zuge der praktischen Umsetzung technische und gesellschaftliche Fragestellungen sowie Werte und Normen nicht getrennt voneinander betrachtet werden können. Das Verhältnis von Empirie und normativer Orientierung wurde zudem im Rahmen der Lehrer(innen)bildung aufgegriffen und vor dem Hintergrund des im sogenannten „Beutelsbacher Konsens" beschriebenen ideologischen Überwältigungsverbots ${ }^{18}$ diskutiert. Die Frage war, inwiefern die Vermittlung der Zustimmung zu Zielen einer Nachhaltigen Entwicklung zu einer Überforderung von Schüler(inne)n führen könnte und welche Rolle dabei die Lehrpersonen spielen.

17 „Six Thinking Hats“ ist eine systematische Anleitung zur Perspektivenübernahme: Probleme werden von sechs verschiedenen Blickwinkeln aus betrachtet und beschrieben, was insgesamt dazu beiträgt, sie besser zu analysieren und in der Folge möglichst gut zu lösen.

18 Der Beutelsbacher Konsens wurde 1976 auf einer Tagung der „Landeszentrale für politische Bildung Baden-Württemberg“ erarbeitet und stellt heute einen anerkannten Standard Politischer Bildung an allen Schulen dar, der auch außerhalb Deutschlands in vielen Ländern beachtet wird (s. a. https://www.lpb-bw.de/beutelsbacher-konsens. html, zugegriffen am 19.05.2019). Er setzt sich zusammen aus den drei Prinzipien „1. Überwältigungsverbot ... 2. Was in Wissenschaft und Politik kontrovers ist, muss auch im Unterricht kontrovers erscheinen ... 3. Der Schüler muss in die Lage versetzt werden, eine politische Situation und seine eigene Interessenlage zu analysieren“ (Wehling 1977, S. 179f.). Das Überwältigungsverbot lautet wie folgt: „Es ist nicht erlaubt, den Schüler - mit welchen Mitteln auch immer - im Sinne erwünschter Meinungen zu überrumpeln und damit an der, Gewinnung eines selbständigen Urteils` zu hindern. Hier genau verläuft nämlich die Grenze zwischen Politischer Bildung und Indoktrination. Indoktrination aber ist unvereinbar mit der Rolle des Lehrers in einer demokratischen Gesellschaft und der - rundum akzeptierten - Zielvorstellung von der Mündigkeit des Schülers“ (Wehling 1977, S. 179f.). 
Vorweg sei angemerkt, dass die im Kontext der Lehre handelnden Personen sich zwar spezifische Rollen zuschreiben können, diese jedoch von der Rollenerwartung der eingebundenen Akteure abweichen können. Zudem können sich die Rollenzuschreibungen während des Prozesses verändern (s. Beecroft 2019 in diesem Band).

Die sich im Kontext der Lehre ergebenden Expositionen von Lehrenden sind daher insbesondere von Rollenerwartungen v. a. seitens der Lernenden geprägt, weniger von der selbstständigen Zuschreibung durch die Lehrenden selbst. Dadurch können Stereotype an Bedeutung gewinnen, etwa bei der Exposition der Lehrenden durch das Forschungsformat. Die Offenheit für Themen und Ergebnisse sowie eine gesamtheitliche Herangehensweise können den Eindruck verstärken, die Lehrenden müssten Fachexpert(inn)en in (möglichst) vielen Disziplinen sein. Als weitere Exposition für die Lehrenden ist die bereits angesprochene Zusammenführung von empirischen und normativen Aspekten zu sehen (vgl. Potthast 2015). Diese entsteht, weil bei Lernenden oft die Vorstellung vorherrscht, wissenschaftliche Arbeit müsse per se unabhängig oder ,neutral' sein, was in Spannung geraten kann zu einer explizit normen- und wertorientierten transdisziplinären und transformativen Arbeit z. B. im Kontext von Nachhaltiger Entwicklung. Diese Erwartungen können zur Überforderung der Lehrenden führen.

Eine Schutzvorkehrung für die Lehrenden ist die Transparenz des Lehr- ebenso wie des Forschungsformats gegenüber den Studierenden. Bereits zu Beginn einer entsprechenden Lehraktivität kann den Studierenden dargelegt werden, welche Fragestellungen in einem transdisziplinären und transformativen Projekt wie beforscht werden. In diesem Prozess kann auch auf die Besonderheit von transdisziplinärer Forschung als Integrationsforschung eingegangen werden, und dies kann mit den fachlichen Hintergründen der Studierenden verglichen werden. Zudem kann ausdrücklich auf den normativen Rahmen aufmerksam gemacht werden. Etwaigen ,Neutralitätserwartungen' an Wissenschaft seitens der Studierenden kann mit Übungssituationen zum Umgang mit explizit normativen Settings begegnet werden (vgl. Beispiel „Methode der Six Thinking Hats“).

Der Mehrwert der vorliegenden Exposition liegt in der Vorbereitung der Studierenden für die anschließende transdisziplinäre Praxis. Normative Perspektiven spielen hier durchwegs eine wichtige Rolle, dies gilt für die gemeinsame Initiierung der Forschungsfrage ebenso wie für die Erarbeitung neuer Wissensbestände wie auch für die Integration der Ergebnisse in Wissenschaft und Gesellschaft. Darüber hinaus können Studierende für den Umgang mit Werten und Normen, die sich durch die Verknüpfung von Forschung mit gesellschaftlichen Anliegen ergeben, in einem geschützten Umfeld geschult werden. Die Lehrenden wiederum können die Erfahrungen aus solchen Veranstaltungen zur Weiterentwicklung von Lehrkonzepten nutzen, sei es thematisch, wie zum Beispiel die Ausgestaltung des Konzepts der Bildung für Nachhaltige Entwicklung, sei es von solchen, die 
sich generell auf transdisziplinäre sowie transformative Lehre beziehen. Als positiven Nebeneffekt können die Studierenden Einblicke in potenzielle Berufsfelder erhalten. Zudem können die Studierenden lernen, ihre eigene Fachdisziplin und ihre Kompetenzen zu reflektieren.

\subsection{Mitwirkung an transdisziplinären und transformativen Lehrveranstaltungen}

Expositionen können entstehen, wenn Studierende, Praxisakteure und Lehrende gemeinsam transdisziplinäre und transformative Lehraktivitäten mit einem starken Praxisbezug gestalten, beispielsweise im Rahmen von Projektseminaren. Expositionen ergeben sich hier vor allem durch den starken Interventionscharakter, das Betätigungsfeld des Praxisakteurs sowie durch unterschiedliche Rollenerwartungen der Akteure.

\section{Beispiel BaWü-Lab Energielabor Tübingen: Durchführung transdisziplinärer und trans- formativer Lehraktivitäten im Kontext des BaWü-Labs}

Im Kontext des BaWü-Labs Energielabor Tübingen wurden in Verbindung mit dem Forschungsprojekt „Wissenschaftliche Bildung und gesellschaftliche Verantwortung" überfachliche Veranstaltungen mit unterschiedlicher thematischer Ausrichtung im Rahmen des überfachlichen Nachhaltigkeitszertifikats „Studium Oecologicum“ durchgeführt.

Im Zuge von Lehrveranstaltungen mit starkem Projektcharakter exponierten sich die Studierenden beispielsweise durch Interventionen auf dem Campus. Hiervon waren auch rechtliche Fragestellungen betroffen. So musste geklärt werden, ob und unter welchen Bedingungen von den Studierenden eine ,Blumenwiese' aus gebrauchten Pappbechern vor der Bibliothek aufgestellt werden durfte. Dabei waren im Vorfeld Abstimmungen mit dem Eigentümer sowie dem Betreiber notwendig. Beispielsweise musste gewährleistet werden, dass zur Schonung der Bäume naturschutzrechtliche Vorgaben zum Wurzelwerk eingehalten und Fragen der späteren Entsorgung geklärt wurden. In einem anderen Fall musste außerdem sichergestellt werden, dass eine öffentliche Kunstperformance vor Gebäuden nicht gegen den Brandschutz oder ähnliche Bestimmungen verstieß. Neben der beabsichtigten Intervention gegenüber der Öffentlichkeit rief die Aktion auch einzelne für diese Gebäude zuständige Mitarbeitende auf den Plan. Aufgrund der Vorgespräche mit den Studierenden zur Rechtslage sowie zur Einhaltung der notwendigen Vorschriften konnten die Studierenden die Situation vor Ort letztendlich selbstständig bewältigen.

Des Weiteren exponierten sich Praxisakteure als Ansprechpersonen für die Studierenden. Auf der operativen Ebene musste beispielsweise geklärt werden, in welchem Umfang Einzelpersonen aus der Praxis den Studierenden während den Arbeitsphasen zur Verfügung standen und welche Erwartungen erfüllt werden konnten. Nicht zuletzt exponierten sich die Praxisakteure in den Veranstaltungen durch ihre Betätigungsfelder 
außerhalb der Lehrveranstaltung. Beispielsweise nutzten manche Studierende die Gelegenheit, um mit dem Verantwortlichen der Cafeterien intensiv über deren Geschäftspraxis zu diskutieren. Dies bezog sich beispielsweise auf das Angebot an veganen Speisen oder den Umgang mit Lebensmittelabfällen.

Studierende können sich in Form von Projektseminaren mit starkem Praxisbezug auf vielfältige Weise exponieren, beispielsweise gegenüber den Praxisakteuren und gegebenenfalls der Öffentlichkeit, wenn sie Aktivitäten wie Befragungen oder Interventionen durchführen oder wenn sie Forschungsergebnisse präsentieren (und je nachdem auch das übergeordnete Forschungsprojekt repräsentieren). Die Rolle von öffentlichen Interventionen wurde im vorangegangenen Kapitel bereits erläutert, soll vor dem Hintergrund der Lehre jedoch noch einmal aufgegriffen werden, da ordnungsrechtliche oder Haftungsfragen nicht zum Tagesgeschäft von Lehrenden zählen, Studierende aber, die im öffentlichen Raum agieren und sich exponieren, dabei einen rechtssicheren Rahmen benötigen.

Des Weiteren exponieren sich Lehrende in ihrer Funktion als Hauptverantwortliche für die Lehrveranstaltung als auch als transdisziplinäre und transformative Wissenschaftler(innen) gegenüber Studierenden und Praxisakteuren.

Außerdem kann Exposition im Fall von Rollenerwartungen, die an die Praxisakteure gerichtet sind, auftreten. Sie können eher operative Dinge betreffen, wie nicht erfüllbare Erwartungen seitens der Studierenden, was zeitliche Ressourcen der Praxisakteure angeht, es können aber auch sehr grundsätzlich Dinge betroffen sein, wenn es etwa um Eingriffe in und Erwartungen an die Arbeit von Praxisakteuren geht. Praxisakteure exponieren sich also möglicherweise während der Veranstaltungen durch ihr Betätigungsfeld.

Eine Schutzvorkehrung für die Studierenden können Lehrende dadurch schaffen, dass sowohl im Vorfeld als auch im Prozess rechtliche Fragestellungen mit den Verantwortlichen geklärt werden. Dies sind hier beispielsweise Gespräche mit Eigentümer(inne)n, Hausmeister(inne)n von Gebäuden oder dem Ordnungsamt. Zudem können in diesem Rahmen die Studierenden für rechtliche Belange sensibilisiert werden. Der zeitliche Aufwand, der für die Klärung rechtlicher Sachverhalte gegebenenfalls benötigt wird, ist dann bereits während der Konzeptionsphase der Lehrveranstaltung zu berücksichtigen. Eine Schutzvorkehrung für Lehrende und Studierende stellt die bewusste Auswahl der Praxisakteure vor der eigentlichen Veranstaltung dar. Die Lehrenden sollten sich im Vorfeld Fragen stellen wie: Welche Erfahrungen mit Expositionsbezug haben wir bereits mit den Praxisakteuren in der Vergangenheit gemacht? Wurden gemeinsame öffentliche Veranstaltungen durchgeführt? Welche positiven oder negativen Positionen haben die infrage kommenden Akteure gegenüber dem Forschungsprojekt? Eine Schutzvorkehrung für die Praxisakteure stellt deren Einbindung während der Konzeption der Lehrveranstaltung dar. Die Vorgespräche können im Vorfeld und 
im kleinen Rahmen ohne Studierende stattfinden. Hier ist zu klären, zu welchen Zeiten die Praxisakteure an der Lehrveranstaltung teilnehmen können, und in welchen Phasen bei Bedarf während der Veranstaltung mit ihnen über den laufenden Prozess diskutiert wird, etwa um unerwünschte Überraschungen gegen Ende zu vermeiden. Die Einbindung von Praxisakteuren in die Konzeption kann auch dazu beitragen, Zielsetzung, Interessen und Erwartungen dieser Akteure bereits im Vorfeld zu diskutieren. Dies ist vor allem dann relevant als Schutzvorkehrung, wenn die Studierenden öffentliche Interventionen realisieren, die die Praxisakteure direkt betreffen. Was die Exposition der Praxisakteure durch deren Betätigungsfeld angeht, ist es Aufgabe der Lehrenden, während der Veranstaltung für Schutz zu sorgen, kritische Konstellationen zu erkennen, zu moderieren und wenn nötig in Diskussionen einzugreifen. Um sich hierauf vorzubereiten, können die Lehrenden bereits bei den Vorgesprächen mit den Praxisakteuren auf mögliche Rollenbilder und Kritikpunkte eingehen.

Ein Mehrwert, den transdisziplinäre und transformative Lehr- und Lernformate haben, besteht in der Durchführung von Interventionen, da etwa die Einbindung weiterer Akteure möglich wird. Ein weiterer Mehrwert liegt in der Erschließung neuer gesellschaftlich relevanter Themen für die Forschung. Zum Ersten können Studierende zusätzliche Kompetenzen und Arbeitskraft einbringen, zum Zweiten haben diese aufgrund ihrer persönlichen Rolle möglicherweise Zugang zu weiteren Akteuren, was den Handlungsrahmen erweitert. Lehrveranstaltungen können so auch Testfelder für die weitere transdisziplinäre und transformative Arbeit sein: Formen der Intervention können ausprobiert oder Hinweise zu neuen Handlungsfeldern gewonnen werden, die im späteren Verlauf eines Forschungsprojekts weiterverfolgt werden können. Ein möglicher positiver Effekt kann zudem sein, dass Studierende für die Komplexität sozio-technischer Themen sensibilisiert werden und institutionelle sowie rechtliche Belange mitdenken.

Durch die entsprechenden Schutzvorkehrungen kann das Vertrauensverhältnis zwischen den unterschiedlichen Akteuren verbessert werden. Dies ermöglicht es beispielsweise den Studierenden, tiefere Einblicke in das Betätigungsfeld der Praxisakteure zu erhalten. Als weiterer positiver Effekt für die Praxisakteure kann die Unterstützung für deren Vorhaben und Interessen gesehen werden. Hier ergeben sich auch Querbezüge zum Lehrformat Service Learning ${ }^{19}$, bei welchem Studierende gemeinwohlorientierte Akteure bei deren Arbeit unterstützen und sich durch die Akteurskonstellation ähnliche Fragen zu Exposition und Schutzvorkehrung ergeben können (vgl. Albiez und Potthast 2018).

19 ,Service Learning - Lernen durch Engagement ist eine Unterrichtsmethode, die gesellschaftliches Engagement von Schülerinnen und Schülern mit fachlichem Lernen im Unterricht verbindet" (Seifert und Zentner 2010, S. 3). In Deutschland wurde die Methode zunächst von Schulen adaptiert und wird inzwischen auch an verschiedenen Universitäten vermehrt angeboten. 


\section{Schlussreflexionen}

In den vorausgegangenen Kapiteln wurde anhand von Beispielen aus verschiedenen BaWü-Labs aufgezeigt, dass sich Akteure im Rahmen von transdisziplinären (und transformativen) Forschungsprojekten in für sie ungewohnten Situationen, ausgelöst durch ungewohnte Aufmerksamkeit und/oder Tätigkeiten, wiederfinden können. Für diese Situationen wurde der Begriff der Exposition eingeführt. Verschiedene Formen der Exposition wurden dargestellt. So wurde zunächst auf Expositionen eingegangen, die aus dem Forschungsdesign und dem Forschungsgegenstand in BaWü-Labs entstanden waren (Kapitel 2), gefolgt von Expositionen, die aus einer verstärkten Öffentlichkeitsarbeit resultierten (Kapitel 3). Daran schlossen sich Expositionen an, deren Ursachen in der Durchführung von Öffentlichen Interventionen lagen (Kapitel 4), um abschließend jene Exposition zu beleuchten, die sich durch die Integration von Lehre in das Forschungsprojekt ergaben (Kapitel 5).

Wie in Kapitel 1 eingeführt, stellt die Reflexion über Expositionen einen anwendungsorientierten, ergänzenden Zugang zu etablierten Ansätzen der Forschung über transdisziplinäre und transformative Forschung dar. Der Schwerpunkt des Beitrags liegt in der Darstellung von verschiedenen Expositionen und möglichen Strategien, mit diesen umzugehen. Im Folgenden wird dargelegt, wie der Zugang über Expositionen in transdisziplinären (und transformativen) Forschungsprojekten zum Tragen kommen kann. Des Weiteren werden Möglichkeiten zur Weiterentwicklung dieses Zugangs aufgezeigt und punktuelle Verknüpfungen zu etablierten Forschungsansätze herausgearbeitet.

\subsection{Expositionen bei Planung und Durchführung eines transdisziplinären und transformativen Forschungsprojekts: die Anwendung}

Den einzelnen Akteuren bietet die Reflexion über Expositionen eine Hilfestellung, um sich auf ihr Mitwirken in einem Forschungsprojekt vorzubereiten. Dazu empfiehlt es sich, die in Kapitel 1 genannten Fragen von einem persönlichen Standpunkt und der jeweiligen spezifischen Situation aus betrachtet anzuwenden. Insbesondere eine bewusste Auseinandersetzung mit bevorstehenden, ungewohnten Tätigkeiten (im Gegensatz zur üblichen Arbeitsroutine) und mit ungewohnter Aufmerksamkeit kann helfen, Expositionen im Vorhinein zu erkennen und sich entsprechend darauf vorzubereiten. Das frühzeitige Erkennen des Mehrwerts von Expositionen und positiven Effekten von Schutzvorkehrungen kann ein Motivationsfaktor für die Arbeit im Forschungsprojekt sein. Beispielsweise kann die Erkenntnis, dass eine zusätzlich notwendige Schulung längerfristig eine (vielleicht schon seit langem angestrebte) Zusatzqualifikation bringt, die Einsatzbereitschaft 
für das Forschungsprojekt fördern. Aber auch während der Projektlaufzeit oder im Nachgang kann eine solche Reflexion helfen, den individuellen Lernprozess zu unterstützen.

Auf der Ebene von Projektleitung und -management hilft eine vorausschauende Reflexion anhand der in Kapitel 1 dargelegten Fragen zu Expositionen, um kritische Situationen, die durch Expositionen ausgelöst werden könnten, zu antizipieren, die Ursachen zu analysieren und sowohl in der Konzeptionsphase als auch bei Zwischenevaluationen zielgerichtete Strategien zu entwickeln. Dabei sollte der Aspekt der Exposition von Seiten aller Akteursgruppen beleuchtet werden, damit alle notwendigen Kompetenzen im Projektteam vertreten sind - spätestens nach Durchführung der Schutzvorkehrungen, die der Aneignung von Kompetenzen dienen. So können die Aufgaben entsprechend den Kompetenzen verteilt, Schutzvorkehrungen mit zeitlichem Vorlauf rechtzeitig in die Wege geleitet, ähnliche Schutzvorkehrungen gebündelt und ausreichend finanzielle Ressourcen für Schutzvorkehrungen wie Schulungen oder das ,Einkaufen' spezieller Expertise oder die Integration von weiteren Akteuren ins Team vorgesehen werden. Ebenso können Mehrwert und positive Effekte in der Projektplanung bewusst berücksichtigt werden. Wichtig ist es bei der Reflexion über Expositionen aus Sicht von Projektleitung und -management, die beteiligten Akteure auch als Individuen zu berücksichtigen, da manche Schutzvorkehrungen vom individuellen Wissens- und Erfahrungsschatz abhängen. So kann beispielsweise eine Weiterbildung im Bereich Medien zur Durchführung eines medialen Aufrufs für eine(n) spezielle(n) Wissenschaftler(in) überflüssig sein, wenn diese(r) bereits im Rahmen von vorhergehenden Projekten Medienkompetenzen erworben hat.

Bezüglich der zeitlichen Komponente empfehlen die Autor(inn)en, in transdisziplinären (und transformativen) Forschungsprojekten eine vorausschauende Reflexion über Expositionen zu Projektbeginn im Anschluss an eine Akteursanalyse durchzuführen. Diese hat zum Ziel, Akteure sowie deren Interessen und Konflikte zu identifizieren und entsprechende Strategien zu entwickeln (zu Akteursanalyse bei Reallaboren vgl. Eckart et al. 2018). Die gewonnenen Erkenntnisse zu Fragen wie etwa, welche Akteure im Projekt beteiligt sind, welche Relevanz und Gewichtung im Forschungsprojekt sie jeweils einnehmen oder wo mögliche Konfliktlinien verlaufen, können beim Erkennen von Expositionen und beim Abwägen von Schutzvorkehrungen hilfreich sein. Eine Reflexion über mögliche auftretende Expositionen kann auch parallel zur Rollenklärung und -festlegung stattfinden. Eine solche wird von Jahn (2008) als notwendiger Teil interdisziplinärer Integration angesehen. Weiter empfiehlt es sich, die Reflexion im Laufe eines Forschungsprojekts zu wiederholen, um Schutzvorkehrungen anzupassen. Durch die Analyse von antizipierten und eingetretenen Expositionen, deren Mehrwert und den positiven Effekten der Schutzvorkehrungen können zudem Lerneffekte im Projekt erhöht werden. 


\subsection{Expositionen im Kontext transdisziplinärer und transformativer Forschung: ein Ausblick}

Wie im vorhergehenden Abschnitt dargelegt, gibt die Reflexion über Expositionen dem Projektmanagement ein Mittel an die Hand, um kritische Situationen, verursacht durch ungewohnte Tätigkeiten und/oder ungewohnte Aufmerksamkeit, antizipieren und entsprechende Schutzvorkehrungen entwickeln zu können. Eine mögliche Weiterentwicklung des im vorliegenden Beitrag dargelegten Zugangs liegt daher in einer Untersuchung, bezogen auf welche Managementansätze für transdisziplinäre (und transformative) Forschungsprojekte die Reflexion über Expositionen in welcher Art und Weise einen Beitrag leisten kann zur Weiterentwicklung beziehungweise zu welchen Managementansätzen diese Reflexion eine sinnvolle Ergänzung darstellt. Als ein mögliches Beispiel seien hier Schophaus et al. (2004) mit ihrem Ansatz des transdisziplinären Kooperationsmanagements genannt. Sie legen einen Schwerpunkt auf den Kooperationscharakter also die Schnittstellen zwischen Akteuren - und geben Handreichungen, wie mit diesen umgegangen werden kann. Hierbei könnte die Betrachtung von Expositionen neue Erkenntnisse bringen, da sie beleuchtet, wo und wie an den Schnittstellen durch ungewohnte Tätigkeiten und/oder Aufmerksamkeit besondere Herausforderungen ausgelöst werden.

Dass eine vertiefte Auseinandersetzung mit dem Thema der Exposition auf der Ebene des Forschungsprojektmanagements zu einem produktiven Forschungsverlauf beitragen kann, lassen auch Erkenntnisse aus der Analyse von Prozessabläufen schließen. So erläutert Jahn (2008), dass das Ausbalancieren von Spannungen entscheidend den Ertrag eines Forschungsprojekts beeinflusst. Ausgehend davon, dass Expositionen Teil der angesprochenen Spannungen sind, bedeutet der Umkehrschluss, dass der bewusste Umgang mit Expositionen für die Ausbalancierung von Spannungen und somit den Projektertrag wichtig sein kann.

Es zeigen sich zudem Anknüpfungspunkte an Konstellationsfragen, wie sie Defila et al. formulieren: „Praxispartner und Forschende bilden eine mehr oder weniger geschlossene Gesellschaft" (2016, S. 58). Hier wäre die Frage nach Expositionen einerseits so zu verstehen, dass ausgehend davon, dass ein transdisziplinäres Team von außen als hermetisch geschlossener Expert(inn)enzirkel wahrgenommen werden könnte, geprüft wird, welche Exposition dies für wen verursachen kann. Andererseits wäre zu fragen, ob nicht eine solche Geschlossenheit auch Schutz vor Exposition Einzelner bietet.

In diesem Beitrag steht der Unterschied zwischen transdisziplinärer und transformativer Forschung nicht im Vordergrund der Expositions-Analysen. Es wäre genauer zu untersuchen, ob sich Forschende insgesamt weniger exponieren, die vermeintlich ,nur' ein Praxisproblem in transdisziplinären Teams bearbeiten, als diejenigen, die explizit und programmatisch damit auch Wissenschaft und Gesell- 
schaft maßgeblich verändern, also transformieren wollen. Für die Frage des Auftretens in der Öffentlichkeit ist diese Unterscheidung möglicherweise nicht besonders relevant, aber innerhalb der Wissenschaftsgemeinschaft hat die Diskussion um transformative Forschung erhebliche Wellen geschlagen (initiiert durch Strohschneider 2014), so dass hier Exposition zu erwarten ist. Dies zeigt sich ansatzweise in der Exposition, die durch transdisziplinäre (und transformative) Lehre entsteht (s. Kapitel 5).

Die Reflexion über Expositionen kann auch als Teilaspekt in die Evaluierung von transdisziplinären (und transformativen) Forschungsprojekten und die Qualitätssicherung für solche Projekte integriert werden. Im Diskurs über Qualitätskriterien und Evaluierung interdisziplinärer, transdisziplinärer und transformativer Forschungsprojekte gibt es Vertreter(innen), die die Relevanz von Lernen und Coachen im Gegensatz zu einem reinen beurteilenden Zugang betonen. So streicht Klein (2006) den Wert eines ,coaching model“" hervor (S. 78), und Bergmann et al. (2005) schlagen eine diskursive, formative Evaluation vor. In solchen diskursiven und coachenden Evaluationsprozessen kann die Analyse von Expositionen und des Umgangs damit zum Lernprozess beitragen, indem besondere Herausforderungen in Form von Ungewohntem erkannt und geplante, durchgeführte und mögliche Strategien des Umgangs reflektiert werden. Des Weiteren kann die Analyse von Expositionen dazu beitragen, die notwendigen Grundlagen für Lernprozesse im Team zu schaffen, da sie machtbedingte Abhängigkeiten („Wer exponiert sich gegenüber wem?“) aufzeigen kann. Machtgefüge haben laut Jahn et al. (2012) einen wichtigen Einfluss auf den wechselseitigen Lernprozess. Schutzvorkehrungen könnten darüber hinaus zur Abdeckung von Lücken in der Qualitätssicherung dienen, wenn zum Beispiel zusätzliche Fortbildungen gewährleisten, dass die notwendige Expertise im Team gegeben ist. Wichtig scheint eine solche Untersuchung auch in Anbetracht von kritischen Stimmen, die in der Forderung nach umfassender, angleichender Kompetenzerweiterung aller Akteure keine Lösung sehen: „Der Umgang mit vorhandenen Ausgangs-, Qualifikationsund Funktionsunterschieden im Kooperationsprozess erwies sich allerdings [...] als kompliziert. Der Anspruch der gleichberechtigten Kooperation scheint dazu zu verleiten, bei Forschern und Praktikern die gleichen Kompetenzen vorauszusetzen oder den Erwerb derselben zum Ziel zu erheben“" (Prengel et al. 2008, S. 186).

Im vorliegenden Beitrag wurden Expositionen ausschließlich anhand von Beispielen aus BaWü-Labs analysiert und dargelegt. Bei einer Analyse von Expositionen im Rahmen von anderen transdisziplinären (und transformativen) Forschungsformaten müssen deren gegebenenfalls anderen Rahmenbedingungen und Grundvoraussetzungen berücksichtigt werden. Diese können Einfluss auf Ursachen, Auswirkungen und Häufigkeit von Expositionen haben. So spielt zum Beispiel der Fördergeber eine wichtige Rolle in der im vorhergehenden Absatz 
bereits angesprochenen Machtstruktur. Die vorgegebene normative Zielrichtung hat ebenfalls Auswirkungen auf die Ursachen, negativen Folgen und den Mehrwert von Expositionen. Eine mögliche Weiterentwicklung wäre daher eine vergleichende Analyse von Expositionen in verschiedenen transdisziplinären (und transformativen) Forschungsformaten unter Berücksichtigung von Rahmenbedingungen der Förderung. Ziel wäre, systematisches Wissen zu Interdependenzen zwischen Rahmenbedingungen, Ursachen, Auswirkungen und Häufigkeit von Expositionen in transformativen (und transdisziplinären) Forschungsprojekten aufzubauen. Expositionen könnten so systematisch erfasst, analysiert und zu einer Datenbank ausgebaut werden. Eine solche Datenbank könnte dazu beitragen, den Aufbau sogenannter „transformative Literacy“ (Schneidewind 2013) voranzutreiben.

Eine weitere Richtung wäre eine Analyse, ob gewisse Methoden in der transdisziplinären (und transformativen) Forschung mehr Expositionen als andere mit sich bringen und wie verhindert werden kann, dass sich dies negativ auf die Prozessqualität auswirkt: „Dienlicher als eine Debatte über die Neuartigkeit der Methoden, die bei transdisziplinärer (und transformativer) Forschung eingesetzt werden, scheint daher eine Auseinandersetzung mit Fragen wie der, wofür sich welche Methode unter welchen Bedingungen eignet, wie sichergestellt werden kann, dass bei der Umsetzung von Methoden die erforderliche Qualität der Prozesse und Ergebnisse erreicht wird, [...]“" (Defila und Di Giulio 2018c, S. 23). Es wäre zu untersuchen, ob eine Reflexion über die zur Wahl stehenden Methoden anhand von Expositionen hierzu beitragen kann. Dies könnte beispielsweise helfen, eine Methode zu wählen, die im Vergleich zu anderen bei gleichem Ertrag mit weniger Expositionen und somit weniger Aufwand für Schutzvorkehrungen einhergeht.

Schließlich möchten die Autor(inn)en eine Untersuchung der Überlagerung und Akkumulation von Expositionen sowie möglicher Strategien der Entzerrung und Resistenz gegen diese anregen. Der vorliegende Beitrag konzentriert sich darauf, einzelne Expositionen möglichst isoliert darzustellen, um die gesammelten Erfahrungen entsprechend konsequent analysieren zu können und das Thema greifbar zu vermitteln. Wie so oft ist die Realität aber komplexer und es kann zu Überlagerungen oder Wechselwirkungen zwischen verschiedenen Expositionen kommen, welche noch einer zusätzlichen Betrachtung bedürfen. Als Beispiel sei an dieser Stelle an die Darlegung in Abschnitt 2.2 erinnert, in der die Schutzvorkehrung für die Praxisakteure die Exposition für die Wissenschaftler(innen) darstellt. 


\subsection{Schutz in der Exposition, Schutz für die Exposition}

Im vorliegenden Beitrag wurde deutlich, dass sich die Beschäftigung mit Exposition lohnt: Die Erfahrungen der BaWü-Labs haben gezeigt, dass Exposition ein konstitutives Merkmal von transdisziplinärer und transformativer Forschung ist. Gleichzeitig können sich aus den Expositionen direkter Mehrwert und/oder aus den Schutzvorkehrungen positive Effekte ergeben, die sich für das Projekt ausschöpfen lassen. So bestehen, zusammengefasst, der Mehrwert der Expositionen, die sich durch das Forschungsdesign und den Forschungsgegenstand ergeben, sowie die positiven Effekte der dazu passenden Schutzvorkehrungen vor allem in der Entwicklung eines gegenseitigen Verständnisses der Akteure, in der Möglichkeit, verhärtete Konflikte aufzulösen, und in der leichteren Verständlichkeit von Forschung (s. Kapitel 2). Durch Öffentlichkeitsarbeit verursachte Expositionen und die dazu passenden Schutzvorkehrungen wiederum können durch eine breite öffentliche Präsenz und damit einhergehende größere Bekanntheit zur Verstetigung beitragen sowie bei der Gewinnung neuer (und unerwarteter) Akteure hilfreich sein (s. Kapitel 3). Expositionen im Rahmen von öffentlichen Interventionen und die passenden Schutzvorkehrungen können Mehrwert und positive Effekte mit sich bringen, indem sie das Forschungsthema in die Bevölkerung tragen und somit breite transformative Prozesse unterstützen (s. Kapitel 4), jene, die durch die Einbindung von Lehre in das Forschungsprojekt verursacht werden, können Praxiserfahrungen für Studierende, Unterstützung von Praxisakteuren durch Studierende sowie weitere Möglichkeiten zum Experimentieren für das Projekt mit sich bringen (s. Kapitel 5).

Ziel einer vorausschauenden Reflexion über Expositionen ist es, ausreichend Schutz in der Exposition zu bieten, um negativen Auswirkungen von Expositionen zu begegnen und den damit verbundenen Mehrwert nutzbar zu machen. Eine mögliche, grundsätzliche Form von Schutzvorkehrung können der bewusste, offene Umgang mit Fehlern und eine entsprechende projektexterne Kommunikation sein, bei der auch über Ergebnisse berichtet wird, die aus Sicht der Beteiligten nicht wie gewünscht verliefen. Schließlich und endlich ist anzumerken, dass das Forschungsformat Reallabor beziehungsweise die Förderung von transdisziplinären (und transformativen) Projekten per se Schutz für die Forschung und für die beteiligten Personen bietet, weil diese Formate damit von den Mittelgebern legitimiert werden. Eine solche Förderung gemeinsam mit entsprechenden Schutzvorkehrungen bieten also im Allgemeinen und im Speziellen Schutz für die Exposition. 


\section{Dank}

Die Autor(inn)en danken Ludger Eltrop für seinen inhaltlichen Input bei der Erstellung des Beitrags und Fabian Dembski, Jochen Eckart, Thorsten Erl, Monika Gonser, Elke Häußler und Jan Riel für die Diskussion über eine frühere Textfassung im Rahmen des internen Reviews. Besonderer Dank gebührt Thomas Becker (Freies Lastenrad Stuttgart), Sarah Behrens (ABK Stuttgart), Timo Dreher (BUND Neckar-Alb), Frank Gwildis (Sachgebiet Stadtentwicklungsplanung der Stadt Stuttgart), Johannes Heynold (JoHey - Studio für Dialog im Raum), Barbara Lupp (BUND Neckar-Alb), Ina Westheiden (ABK Stuttgart) und einem anonymen Praxispartner von STADT-RAUM-BILDUNG für die Feedbacks aus ihrer Sicht als Praxisakteure. Herzlichen Dank an Matthias Rudolph und Juliane Windbiel für die Recherche und Bereitstellung des Bildmaterials sowie an Constantin Hörburger für die Recherche von Unterlagen (alle drei ABK Stuttgart). Insbesondere möchten die Autor(inn)en auch den beiden anonymen externen Gutachter(inne)n für die konkreten Hinweise zur Verbesserung des Textes danken. Schließlich danken sie den beiden Herausgebenden, Rico Defila und Antonietta Di Giulio, für ihre Rückmeldungen zum Text.

\section{Literatur}

Albiez, M., König, A., \& Potthast, T. (2018). Transdisziplinarität und Bildung für Nachhaltige Entwicklung in der Lehre an der Universität Tübingen: Konzeptionelle Fragen mit Bezug auf Lehraktivitäten des „Energielabors Tübingen“. In W. Leal Filho (Hrsg.), Nachhaltigkeit in der Lehre. Eine Herausforderung für Hochschulen (S. 189-206). Berlin, Heidelberg: Springer Spektrum.

Albiez, M., \& Potthast, T. (2018). Gemeinsam lernen, forschen, engagieren. Alles eins? In: N. Neuber, W. Paravicini \& M. Stein (Hrsg.), Forschendes Lernen. The Wider View. Eine Tagung des Zentrums für Lehrerbildung der Westfälischen Wilhelms-Universität Münster vom 25. bis 27.09.2017 (Schriften zur Allgemeinen Hochschuldidaktik) (S. 139-142). Münster: WTM-Verlag.

Beecroft, R. (2019). Das „Transformative Projektseminar“ - didaktische Ansätze und methodische Umsetzung. In R. Defila \& A. Di Giulio (Hrsg.), Transdisziplinär und transformativ forschen, Band 2. Eine Methodensammlung (S. 293-337). Wiesbaden: Springer VS.

Bergmann, M., Brohmann, B., Hoffmann, E., Loibl, M. C., Rehaag, R., Schramm, E., \& Voss, J.-P. (2005). Qualitätskriterien transdisziplinärer Forschung. Ein Leitfaden für die formative Evaluation von Forschungsprojekten. Frankfurt a. M.: Institut für sozialökologische Forschung. https://www.isoe.de/ftp/evalunet_leitfaden.pdf. Zugegriffen am 15.04.2019.

Bergmann, M., Jahn, T., Knobloch, T., Krohn, W., Pohl, C., \& Schramm, E. (2010). Methoden transdisziplinärer Forschung. Ein Überblick mit Anwendungsbeispielen. Frankfurt a. M., New York: Campus. 
Boix Mansilla, V., Feller, I., \& Gardner, H. (2006). Quality assessment in interdisciplinary research and education. Research evaluation, 15 (1), (S. 69-74). doi: 10.3152/1471544 06781776057.

De Bono, E. (1999). Six thinking hats. 1. Back Bay Paperback Aufl., überarbeitet und aktualisiert. Boston: Back Bay Books.

Defila, R., \& Di Giulio, A. (1999). Transdisziplinarität evaluieren - aber wie? Evaluationskriterien für inter- und transdisziplinäre Forschung. Panorama. Sondernummer 1999. https://forschungsverbundmanagement.net/publikationen/Sondernummer.Pano.1. 99.pdf. Zugegriffen am 15.04.2019.

Defila, R., Di Giulio, A., \& Scheuermann, M. (2006). Forschungsverbundmanagement. Handbuch für die Gestaltung inter- und transdisziplinärer Projekte. Zürich: vdf Hochschulverlag an der ETH Zürich.

Defila, R., Di Giulio, A., \& Schäfer, M. (2016). Hotspots der transdisziplinären Kooperation - Ausgangslagen von besonderer Bedeutung. In R. Defila \& A. Di Giulio (Hrsg.), Transdisziplinär forschen - zwischen Ideal und gelebter Praxis. Hotspots, Geschichten, Wirkungen (S. 27-89). Frankfurt a. M., New York: Campus.

Defila, R., \& Di Giulio, A. (2018a). Transdisziplinarität und Reallabore. In Reallabor für nachhaltige Mobilitätskultur Universität Stuttgart (Hrsg.), Stuttgart in Bewegung. Berichte von unterwegs (S. 31-37). Berlin: Jovis. doi: 10.18419/opus-10234.

Defila, R., \& Di Giulio, A. (Hrsg.). (2018b). Transdisziplinär und transformativ forschen. Eine Methodensammlung. Wiesbaden: Springer VS. doi: 10.1007/978-3-658-21530-9.

Defila, R., \& Di Giulio, A. (2018c). Reallabore als Quelle für die Methodik transdisziplinären und transformativen Forschens - eine Einführung. In R. Defila \& A. Di Giulio (Hrsg.), Transdisziplinär und transformativ forschen. Eine Methodensammlung (S. 9-35). Wiesbaden: Springer VS. doi: 10.1007/978-3-658-21530-9_1.

Eckart, J., Ley, A., Häußler, E., \& Erl, Th. (2018). Leitfragen für die Gestaltung von Partizipationsprozessen in Reallaboren. In R. Defila \& A. Di Giulio (Hrsg.), Transdisziplinär und transformativ forschen. Eine Methodensammlung (S. 105-135). Wiesbaden: Springer VS. doi: 10.1007/978-3-658-21530-9_6.

Gonser, M., Eckart, J., Eller, C., Köglberger, K., Häußler, E., \& Piontek F. M. (2019). Unterschiedliche Handlungslogiken in transdisziplinären und transformativen Forschungsprojekten - Welche Risikokulturen entwickeln sich daraus und wie lassen sie sich konstruktiv einbinden? In R. Defila \& A. Di Giulio (Hrsg.), Transdisziplinär und transformativ forschen, Band 2. Eine Methodensammlung (S. 39-83). Wiesbaden: Springer VS.

Groß, M., Hoffmann-Riem, H., \& Krohn, W. (2005). Realexperimente. Ökologische Gestaltungsprozesse in der Wissensgesellschaft. Bielefeld: transcript.

Jahn, T. (2008). Transdisziplinarität in der Forschungspraxis. In M. Bergmann \& E. Schramm (Hrsg.), Transdisziplinäre Forschung. Integrative Forschungsprozesse verstehen und bewerten (S. 21-37). Frankfurt a. M., New York: Campus.

Jahn, T., Bergmann, M., \& Keil, F. (2012). Transdisciplinarity: Between mainstreaming and marginalization. Ecological Economics, 79, (S. 1-10). doi: 10.1016/j.ecolecon. 2012.04.017. 
Klein, J. T. (2006). Afterword: the emergent literature on interdisciplinary and transdisciplinary research evaluation. Ecological Economics, 15 (1), (S. 75-80). doi: 10.3152/ 147154406781776011.

Lang, D. J., Wiek, A., Bergmann, M., Stauffacher, M., Martens, P., Moll, P., Swilling, M., \& Thomas, C. J. (2012). Transdisciplinary research in sustainability science: practice, principles, and challenges. Sustainability Science, 7 (Supplement 1), (S. 25-43). doi: 10.1007/s11625-011-0149-x.

Lazarova, K., Helfenstein, B. S., Dietz, R., \& Alcántara, S. (2018). Parklets für Stuttgart. Ein Realexperiment. Stuttgart: Reallabor für nachhaltige Mobilitätskultur Universität Stuttgart. http://www.r-n-m.net/wp-content/uploads/2018/03/Parklets-fuer-Stuttgart_ web.pdf. Zugegriffen am 27.02.2019.

McDonald, D., Bammer, G., \& Dean, P. (2009). Research integration using dialogue methods. Canberra, Australia: ANU E Press, The Australian National University. https://press-files.anu.edu.au/downloads/press/p60381/pdf/book.pdf. Zugegriffen am 15.04.2019.

MWK (Ministerium für Wissenschaft, Forschung und Kunst Baden-Württemberg) (Hrsg.). (2013). Wissenschaft für Nachhaltigkeit. Herausforderung und Chance für das badenwürttembergische Wissenschaftssystem. Stuttgart. https:/www.baden-wuerttemberg.de/ fileadmin/redaktion/dateien/PDF/Broschüre_Wissenschaft_für_Nachhaltigkeit.pdf. Zugegriffen am 19.05.2019.

Pohl, C., \& Hirsch Hadorn, G. (2006). Gestaltungsprinzipien für die transdisziplinäre Forschung. Ein Beitrag des td-net. München: oekom.

Potthast, T. (2015). Ethics and Sustainability Science beyond Hume, Moore and Weber. Taking Epistemic-Moral Hybrids Seriously. In S. Meisch, J. Lundershausen, L. Bossert \& M. Rockoff (Hrsg.), Ethics of Science in the Research for Sustainable Development (S. 129-152). Baden-Baden: Nomos.

Prengel, A., Heinzel, F., \& Carle, U. (2008). Methoden der Handlungs-, Praxis- und Evaluationsforschung. In W. Helsper \& J. Böhme (Hrsg.), Handbuch der Schulforschung (S. 181-197). Wiesbaden: Springer VS.

Puttrowait, E., Dietz, R., Gantert, M., \& Heynold, J. (2018). Der Weg zum Realexperiment - Schlüsselakteure identifizieren, Kooperationsstrukturen aufbauen, Projektideen auswählen. In R. Defila \& A. Di Giulio (Hrsg.), Transdisziplinär und transformativ forschen. Eine Methodensammlung (S. 195-232). Wiesbaden: Springer VS. doi: 10. 1007/978-3-658-21530-9_11.

RLSS (Reallabor Space Sharing, ABK Stuttgart) (Hrsg.). (2018). One Space Fits All Space Sharing Report. Erfahrungsbericht und Empfehlungen aus zwei Jahren Betriebserfahrung des Reallabor Space Sharing-Pilotprojekts. Stuttgart: Staatliche Akademie der Bildenden Künste.

San Francisco Planning Department (2018). San Francisco - Parklet Manual. San Francisco: City of San Francisco. http://groundplaysf.org/wp-content/uploads/Parklet_Manual_ 2018-FINAL_upload.pdf. Zugegriffen am 21.05.2019.

Schäpke, N., Stelzer, F., Bergmann, M., Singer-Brodowski, M., Wanner, M., Caniglia G., \& Lang, D. (2017). Reallabore im Kontext transformativer Forschung. Ansatzpunkte zur Konzeption und Einbettung in den internationalen Forschungsstand. (No. 1/2017). 
Leuphana Universität Lüneburg, Institut für Ethik und Transdisziplinäre Nachhaltigkeitsforschung. http://hdl.handle.net/10419/168596. Zugegriffen am 20.05.2019.

Schneidewind, U. (2013). Transformative Literacy. Gesellschaftliche Veränderungsprozesse verstehen und gestalten. GAIA, 22 (2), (S. 82-86).

Schophaus, M., Schön, S., \& Dienel, H.-L. (Hrsg.). (2004). Transdisziplinäres Kooperationsmanagement. Neue Wege in der Zusammenarbeit zwischen Wissenschaft und Gesellschaft. München: oekom.

Seebacher, A., Alcántara, S., \& Quint, A. (2018). Akteure in Reallaboren - Reallabore als Akteure. In R. Defila \& A. Di Giulio (Hrsg.), Transdisziplinär und transformativ forschen. Eine Methodensammlung (S. 155-159). Wiesbaden: Springer VS. doi: 10. 1007/978-3-658-21530-9_9.

Seifert, A., \& Zentner, S. (2010). Service-Learning - Lernen durch Engagement: Methode, Qualität, Beispiele und ausgewählte Schwerpunkte. Eine Publikation des Netzwerks Lernen durch Engagement. Weinheim: Freudenberg Stiftung. http://buendnis. augsburg.de/fileadmin/buendnis-aug/dat/2_projekte/tu_was/3Pub_Wissen_LdE.pdf. Zugegriffen am 27.02.2019.

Strohschneider, P. (2014). Zur Politik der Transformativen Wissenschaft. In: A. Brodocz, D. Herrmann, R. Schmidt, D. Schulz \& J. Schulze Wessel (Hrsg.), Die Verfassung des Politischen (S. 175-192). Wiesbaden: Springer VS. doi: 10.1007/978-3-658-04784-9_10.

Wehling, H.-G. (1977). Konsens à la Beutelsbach? Nachlese zu einem Expertengespräch. In S. Schiele \& H. Schneider (Hrsg.), Das Konsensproblem in der politischen Bildung (S. 173-184). Stuttgart: Ernst Klett.

Open Access Dieses Kapitel wird unter der Creative Commons Namensnennung 4.0 International Lizenz (http://creativecommons.org/licenses/by/4.0/deed.de) veröffentlicht, welche die Nutzung, Vervielfältigung, Bearbeitung, Verbreitung und Wiedergabe in jeglichem Medium und Format erlaubt, sofern Sie den/die ursprünglichen Autor(en) und die Quelle ordnungsgemäß nennen, einen Link zur Creative Commons Lizenz beifügen und angeben, ob Änderungen vorgenommen wurden.

Die in diesem Kapitel enthaltenen Bilder und sonstiges Drittmaterial unterliegen ebenfalls der genannten Creative Commons Lizenz, sofern sich aus der Abbildungslegende nichts anderes ergibt. Sofern das betreffende Material nicht unter der genannten Creative Commons Lizenz steht und die betreffende Handlung nicht nach gesetzlichen Vorschriften erlaubt ist, ist für die oben aufgeführten Weiterverwendungen des Materials die Einwilligung des jeweiligen Rechteinhabers einzuholen.

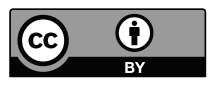

